\title{
A Stochastic Multiplicative Cascade Model for Monitoring Relapse in Mood Disorder Using Wearable Data
}

\author{
Rachel Heath \\ School of Psychological Sciences \\ University of Newcastle, Australia
}

\begin{abstract}
A person's mental health depends on how well they cope with environmental demands from family, work and other potentially stressful situations. We examine the idea that a chronic illness, such as bipolar disorder, can be monitored using a complexity index obtained from a series of behavior measurements, such as activity measured by an ActiWatch and heart-rate recorded by a Fitbit. Low health status is associated with a reduction in complexity. A multiplicative cascade model driven by a Gaussian process produced a multifractal spectrum from which a Health Index could be computed using the Gaussian mean and standard deviation. For a young person diagnosed with bipolar disorder, this Health Index increased from weeks 1 to 9, during which time the person was well, and then decreased rapidly during the next five weeks preceeding hospitalisation for a manic relapse that occurred on week 14 . A statistical change detection procedure indicated a significant decrease in Health Index two weeks before hospitalisation. Similar findings were obtained using heart-rate data from people diagnosed with bipolar disorder, one of whom suffered severe illness and had to withdraw from the experiment, and the other suffered a self-harm incident requiring emergency treatment. Future applications include an on-line monitoring system that allows timely medical intervention to maintain good mental health and lower the need for hospitalisation and emergency care.
\end{abstract}




\section{Introduction}

We explore how complexity measures based on nonlinear technologies can be used to monitor a person's wellness over extended periods of time using proof-of-concept examples from mental health. The fundamental principle is that good health is characterized by a high level of complexity in behavioral and physiological measures recorded over time, whereas an early sign of a progressive decline in health is indicated by a gradual reduction in complexity. Although the available data are somewhat scant, several single-case proof-of-concept studies have indicated that serious relapse in mental health is accompanied by a decrease in complexity of data acquired using wearable devices that measue heart rate and activity. Whereas recently we have shown that a decline in health is indicated by a reduction in sample entropy and permutation entropy (Heath, 2022, 28 February), an alternative approach uses multiplicative cascade models that yield multifractal spectra. When these cascades are driven by a homogeneous Gaussian process, we can estimate the mean and variance that allows computation of an entropy measure derived using classical Shannon information theory techniques.

We begin with some background information on complexity as it relates to mental health. Then we analyse activity data for people diagnosed with bipolar disorder. These analyses show how wearable devices can be used to detect the early signs of a mental illness relapse. By doing so, timely intervention can be implemented to avoid adverse consequences, such as admission to hospital with its associated costs to the person, their family and the community. Other examples use heart rate data obtained from a commercially available device, a Fitbit. In both examples we employ people who have been diagnosed with bipolar disorder, and in an associated experimental study, their matched controls. 


\section{Complexity and Mental Health}

The complexity of mental health is revealed by the complicated network structures that bind the various symptoms people report for mental disorders. Collectives of these symptoms can be linked to offer a new insight into the diagnoses of mental illness. For example, major depression can be characterised by extra morning sleepiness and an increase in weight. These changes are often accompanied by dysregulation of other bodily systems, especially those associated with the inflammatory response (Boschloo, van Borkalo, Rhemtulla, Keyes, Borsboom \& Schoevers, 2015).

Borsboom (2017) proposed a general network model for the dynamics of mental illness resulting from interactions between symptoms and their biological and environmental influences. If the interactions are strong enough, the dynamics become self-sustaining leading to mental illness. For recovery to occur, the original complexity of the person-environmental interaction needs to be restored. One's mental health is determined by the pattern of activation that flows through the symptom network. If persistent clusters evolve, these may be associated with a stable state of mental ill-health. It is the self-sustaining property of the symptom network dynamics that ensures that the illness persists long after the environmental triggers have terminated, as is common in conditions such as post-traumatic stress disorder. This is an example of hysteresis, persistence of the current state of a system without immediate return to previous states. The current state plays an important role in the maintenance of a mental illness, provided the network contains strong connections that reduce its overall ability to adapt appropriately to potentially favorable environmental influences.

Mental health is more likely when the network connections are weak and stable, a state to which the network returns when it has been released from environmental stressors. This is characteristic of resilient people who can readily deal with environmental stressors. 
Vulnerable people, on the other hand, are at risk of returning to a strongly connected symptom state. So weak environmental stressors and weak network connectivity are conducive to good mental health, whereas overly strong associations between these variables can lead to mental illness, a maladaptive person-environment contingency.

Our first case study to illustrate the application of wearable technology to the monitoring of mental illness uses an ActiWatch to monitor a patient's movements 24/7, an example of actigraphy. The use of actigraphy for predicting dynamic fluctuations in potentially psychopathological behaviour may be more generally applicable than appears at first sight. An interesting small-world network analysis of the contagion of all diagnoses contained in DSM-IV (Borsboom, Cramer, Schmittmann, \& Waldorp, 2011) revealed four diagnostic "hubs" (by analogy with airline route networks), insomnia, with 71 connections to other symptoms, psychomotor agitation (68 connections), psychomotor retardation (61 connections) and depressive mood (60 connections). These four diagnostic "hubs" subsume 208 other symptoms in DSM-IV as well as 69 separately described disorders, a massive compression of diagnostic information (see Table 1 in Hu, Scheer, Ivanov, Buijs \& Shea, 2007). All four diagnostic "hubs" can be assessed using actigraphy, whereas heart rate may reflect more general person-environment interactions. Hence the proposed technology presented in this paper may have wide application in the monitoring of substantial aspects of psychopathology.

\section{Physical Activity and Mental Health}

In a study conducted in 1992-1993, regular exercise was reported by about $60 \%$ of a large sample of American adults aged 15 to 54. Although regular exercise was associated with a reduced likelihood of major depression and phobia, there was no significant relation to generalized anxiety (unless comorbid) and bipolar disorder (Goodwin, 2003). Janney, Fagiolini, Swartz, Jakicic, Holleman and Richardson (2014) asked 60 adults diagnosed with 
bipolar disorder to wear an ActiGraph on the hip for seven days, and compared their activity with that of 60 matched controls. The ActiGraph is a device that measures a person's activity, similarly to the ActiWatch that we used in our example application. For these people, most of whom were in a euthymic or well state during the observation period, activity was mostly sedentary $(78 \%)$, this being significantly greater for the bipolar patients than controls.

\section{Activity in People at Risk of Bipolar Disorder}

Bipolar disorder is a severe psychiatric condition with a lifetime prevalence of about $2 \%$ (Merikangas, Jin, He, Kessler, Lee, Sampson, et al., 2011). The disorder is characterised by episodes of severe depression and mania interrupted by well periods of varying duration. Bipolar disorder generates significant morbidity and mortality for the individual, disruption for friends and family, and a significant financial burden due to loss of employment and caring responsibilities by family members. Bipolar disorder, like many other mental illnesses, also incurs a huge cost to health care systems. People diagnosed with bipolar disorder are more likely than those with unipolar depression to develop psychotic and other severely debilitating symptoms that require frequent hospitalisations (Goodwin \& Jamison, 2007).

Although the burden from bipolar depression can be debilitating, manic episodes are the most functionally impairing phase of the disorder, frequently accompanied by psychosis, hyperactivity and sleep disturbance that may require expensive hospitalisation (Grunze, 2010). If a mood episode is identified early, timely intervention for mania may obviate the need for hospitalisation. Perhaps the use of technology can allow people to identify their own early signs of an episode and obtain timely medical assistance themselves. When this is possible, those suffering a manic episode may experience better outcomes (Lam \& Wong, 2005).

Current treatment guidelines for bipolar disorder encourage daily mood monitoring to support early intervention. However, this strategy is not ideal because of its demands on 
people and its unreliability due to a person's possible impaired insight. Research has suggested that 24-hour activity monitoring using a wearable device has the potential to provide automated, objective information about episode precursors (Bullock, Judd \& Murray, 2014).

A characteristic feature of mood disorder is the observed fluctuations in activity levels exhibited by patients as their mood changes. One of the early signs of depression, for example, is a reduction in activity level as the patient withdraws from their usual activities and leads a more sedentary life. On the other hand, incipient mania typically appears as increased physical activity and decreased sleep (Murray, 2010). These changes in the complexity of activity and other measures, such as heart rate in people diagnosed with heart disease, EEG amplitude and frequency for epileptic seizures, and mood rating variability for people with depression are often indicators of a transition to poorer health (Heath, 2004). Indeed, changes in goal-directed activity are now recognised alongside mood changes as a core diagnostic feature for mania (American Psychiatric Association, 2013). So, a reduction in the complexity of activity measures obtained from wearable devices might serve as a precursor for the start of a mental illness relapse

Bullock and Murray (2014) investigated changes in activity and circadian rhythm in people who were either at high or low risk for bipolar disorder. The tendency for people to gravitate towards high or low levels of mood is indicative of a two-dimensional view of depression and mania in people diagnosed with bipolar disorder. This view of mood can be assessed in normal populations using the General Behavior Inventory $(G B I)$ that provides separate measures of mania and depression. The $G B I$ is one of the few instruments that are worth considering for prognostic evaluations of bipolar risk, the total $G B I$ score providing quantitative evidence of a person's vulnerability to bipolar disorder (Ratheesh, Berk, Davey, McGorry \& Cotton, 2015). 
In Bullock and Murray's study, those in the top and bottom $10 \%$ of scores on the $G B I$ were selected for an actigraphy study lasting seven days with 36 people in each group, 75\% of whom were female with a mean age of 22 years. People at risk of bipolar disorder are likely to have unstable circadian rhythms, and their variable lifestyle can lead to variations in their social rhythms. As attitudes towards achievement, dependence and self-control are predictive of relapse, these people also completed the 24-item Dysfunctional Attitudes Scale.

Relative activity during any 24-hour period was measured as the ratio of the difference between activity in the most active 10-hour period and in the least active 5-hour period divided by the total activity in that 24-hour period. An important finding from the study was lower relative activity for those high-risk people with a previous history of a depressive episode, even though there was no difference in overall activity between the two groups. The elevated relative activity in the high-risk group was reflected mostly in greater activity in the evening compared to low-risk subjects. However, it is worth noting that a large national study in the USA found no relationship between bipolar disorder and any variation from the norm in overall physical activity (Cairney, Veldhuizen, Faulkner, Schaffer \& Rodriguez, 2009).

Psychomotor retardation, a dramatic reduction in movement, is common in about $40 \%$ of young people with severe depression. Finazzi, Mesquita, Lopes, Fu, Oliveira and Del Porto (2010) used actigraphy to measure activity in six adolescents, mean age 16 years, who had been diagnosed with depression and offered antidepressant medication. Corroborative information about progress was obtained by weekly scores on the Children's Depression Rating Scale Revised (CDRS-R). Measures of activity over nine weeks were obtained using an ActiWatch. There was a significant negative correlation between the mean activity and depression score over the nine weeks indicating that the measured reduction in depression 
was associated with an increase in mean activity level. This finding was only significant for activity recorded between noon and $6 \mathrm{pm}$.

In the next section, we discuss how information obtained from wearable devices can be used to montitor a person's wellness over time. In these applications we use activity and heart-rate measures to inform a model representing the complex processes that subsume people's transitions from well states to possibly unwell states. We analyse a person's activity in the weeks prior to a manic relapse, and two people's heart rate that served as the precursor to prolonged illness and a serious self-harm event. Statistical decision-making methodologies can then be used in a similar way to industrial quality control to detect the significance of these changes so that the user and nominated others can be advised appropriately.

\section{Multifractal Dynamics of Activity Data in Bipolar Disorder}

Complexity can be defined in terms of the predictability of a sequence of observations of the same phenomenon, such as activity recorded during a two-minute observation period. Throughout any 24-hr period, the activity level fluctuates from one moment to the next, the extent of these fluctuations providing information about the complexity of the associated psychomotor processes. Regular fluctuations indicate a low level of complexity, the least complex example being the regular tick of a clock. As a process becomes more complex its fluctuations become more erratic, an extreme example being random noise. Most physiological systems exhibit behavior somewhere between these two extremes. Importantly, research in several domains has found that it is worthwhile monitoring changes in complexity as significant decreases may suggest the onset of pathology (Heath, 2004).

Some medical disorders are characterized by a reduction in the complexity of at least one associated measurement, such as heart rate in the case of heart dysfunction, EEG amplitude and frequency for epileptic seizures, and mood rating variability for people with unexplained medical symptoms (Burton, Heath, Weller \& Sharpe, 2010) and unipolar 
depression (Heiby, Pagano, Blaine, Nelson \& Heath, 2003). We propose that a similar diagnostic signal based on passive monitoring using a wearable device might be used to detect the imminent onset of a change in health status for people diagnosed with bipolar disorder.

Changes in activity over time in people diagnosed with bipolar disorder are suggested by the recurrent fluctuations in mood from depression, frequently accompanied by low levels of activity, through periods of euthymia and mania that are typically accompanied by increases in activity level (Goodwin \& Jamison, 2007). As indicated in a previous section, actigraphy, which is commonly used to measure sleep and circadian rhythms under naturalistic conditions, records three-dimensional movement using an accelerometer and onboard memory storage (Ancoli-Israel, Cole, Alessi, Chambers, Moorcroft \& Pollak, 2003). The actigraphy data analysed in the following analyses have been described in a case report (Bullock, Judd \& Murray, 2014) that used activity measurements collected by a wrist-worn ActiGraph to monitor the behaviour of a 20-year-old male diagnosed with bipolar disorder type I. The participant was enrolled in a 12-month prospective study of actigraphicallymeasured sleep and circadian function for use as predictors of daily mood in bipolar disorder. For this patient, activity was recorded for 103 days prior to their withdrawal from the study due to hospitalisation for a manic episode.

\section{Log-Log Power Spectrum}

As Delignières, Torre and Lemoine (2011) have shown, the power spectrum relating power to frequency for a time series such as activity can provide useful information about temporal dependencies in the series. When logarithms are taken of both the power and the frequency, the linearity of the log-log function has been associated with long-range correlations, provided confirming evidence is obtained by applying other techniques such as Detrended Fluctuation Analysis (DFA). 
DFA has been used to quantify the fractal nature of a time series. A fractal is a mathematical object that is scale-invariant (Mandelbrot, 1997), so no matter what time scale we choose to measure it, the fractal object always appears the same. In this case, we assume that the mathematical form of activity time series, $A(t)$, does not depend on the time scale used to measure it, so it satisfies the equation $A(c t)=c^{H} A(t)$, where $c$ is a scale constant and $H$ is the Hurst Exponent. The latter is a constant in this monofractal case. This selfsimilarity property, which occurs in many physiological time series such as heart rate, has also been found in actigraphy data, especially when assessed during sleep (Yamahara, Noguchi, Okawa \& Yamada, 2009).

Before applying DFA, the mean activity is subtracted from each activity value. These differences are accumulated and fit by a straight-line to generate prediction errors. The mean fluctuation is obtained by taking the square root of the average squared prediction error. This calculation is performed for nonoverlapping data intervals that increase in length from about 10 up to half the length of the time series. The slope of the linear relationship between the logarithm of the mean fluctuation and the logarithm of the interval length provides an estimate of monofractality known as the Hurst Exponent, a number between 0 and 1.

Knowing the Hurst Exponent is useful because any value less than 0.5 indicates an antipersistent process that corrects itself if the values become too small or too large, whereas a Hurst Exponent greater than 0.5 indicates a persistent process that tends to maintain its current state, increasing, decreasing or remaining relatively constant. By contrast, Gaussian noise is characterized by a Hurst Exponent equal to 0.5, a completely random sequence. As these processes can be represented by a single Hurst Exponent, they are defined as monofractal. 


\section{Multifractal Spectral Analysis of Activity Data}

A multifractal process involves a generalization of DFA to include processes for which the Hurst exponent is no longer constant over all time scales. Such processes can be quite complex. Examples from the physical world include atmospheric turbulence and water flowing over a waterfall. Many people would have experienced the effects of atmospheric turbulence while travelling in an aircraft. The fluctuations in turbulence can be experienced on several time scales, moving up and down in your seat, your coffee level bouncing around and the wing ailerons performing a very complicated dance to keep the aircraft in level flight. Evidence for a multifractal process is obtained when the Hurst exponent is not constant but depends on the fluctuation order, or fractal index (Kelty-Stephen, Palatinus, Salzmann \& Dixon, 2013).

Multifractal data analyses can be performed using Multifractal Detrended Fluctuation Analysis (MFDFA) (Kantelhardt, Zschiegner, Koscielny-Bunde, Bunde, Havlin, \& Stanley, 2002; Ihlen, 2012). The calculations use several data window sizes ranging from 8 to the power of two that is no greater than $20 \%$ of the number of observations in the time series. For example, in the analyses of activity data in which data are accumulated over 10 min periods (1008 observations per week), the maximum window size is the largest integer less than $\log _{2}(1008 / 5)=7.7$, which is 7 . The program returns the fluctuation function variance, the generalized Hurst exponent, the multifractal exponent, and the abscissa and ordinate of the multifractal spectrum (see the Appendix for technical details of this methodology). By fitting a cubic equation to the data samples (cubic detrending), we can effectively control for nonlinear temporal variations, leading to nonstationary parameters that are responsible for variability in activity (Ihlen \& Vereijken, 2013). In the analyses that follow, the results were almost precisely the same irrespective of whether linear, quadratic or cubic detrending was 
applied. So the degree of the equation used for removing trend has no major effect on the results of the analysis.

As Ihlen (2012) has shown, complex fluctuations in time series of various amplitudes can be evaluated by estimating the Generalized Hurst Exponent, $H q$, for various values of $q$, the fractal or singularity index, $-5 \leq q \leq 5$. If $H q$ does not depend on $q$, the time series is monofractal implying that all types of fluctuations, large and small, scale in the same way. This monofractal effect is shown by the red and green horizontal lines for simulated monofractal and Gaussian data, respectively, shown in Figure 1. If $H q$ decreases as $q$ increases, the time series contains fluctuations on many different time scales resulting in it being multifractal. Positive values of $q$ reflect the effect of low frequency temporal fluctuations, whereas negative values result from high frequency fluctuations with small variance. An example of this relationship for a simulated multifractal series is shown by the blue line in Figure 1.

The Hurst Exponent estimated by DFA is the value of $H q$ when $q=2$, a special case of a monofractal, so computing a separate DFA of the data is not necessary. In the following analyses we will concentrate on the multifractal spectrum. The algorithm devised by Ihlen (2012), upon which this analysis is based, incorporates modifications recommended by Kantelhardt et al. (2002) to accommodate instabilities that arise when $q$ is close to 0 or is negative. 


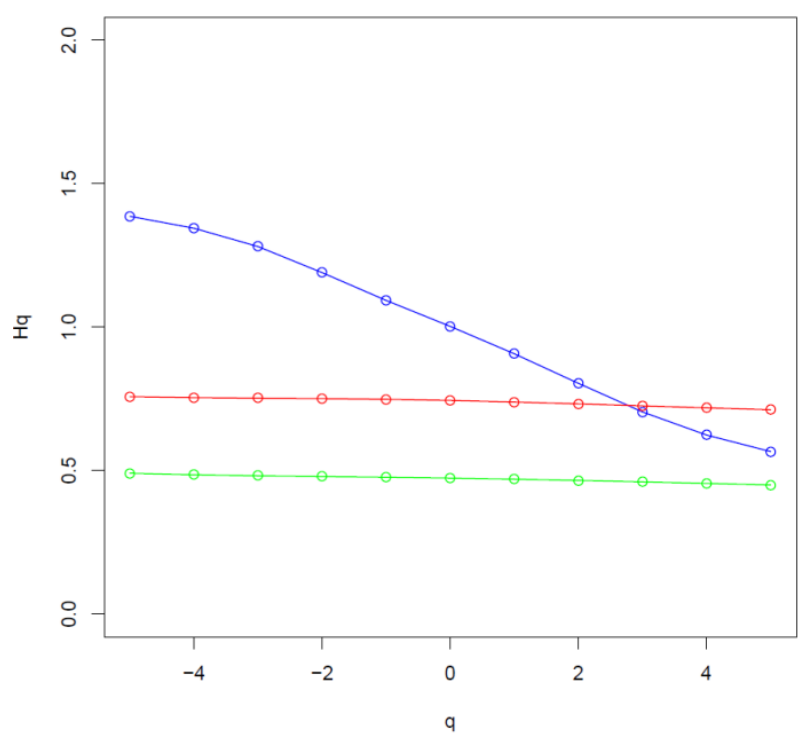

Figure 1. The relationship between the estimated Hurst Exponent, $H q$, and fluctuation function order, $q$, for Gaussian noise (green), and monofractal (red) and multifractal (blue) series obtained by applying the Multifractal Detrended Fluctuation Analysis to data from Ihlen (2012).

The multifractal spectrum relates the multifractal amplitude, $f(\alpha)$, to the fractal index, $\alpha$. For a Gaussian or monofractal series, the multifractal spectrum has a single value of 1 located at $\alpha$ equal to the DFA value, being 0.5 for the Gaussian series and some other number between 0 and 1 for any other monofractal series.

By contrast, the multifractal spectrum for a multifractal series is frequently inverse parabolic in shape with a maximum value of 1 occurring for some value of $\alpha$. As the width of the multifractal spectrum can be defined for any value of $f(\alpha)$, it makes sense to fit the spectrum with a quadratic polynomial and identify the spectrum width with the distance between the roots of this best-fitting quadratic when $f(\alpha)=0$. Figure 2 shows predicted multifractal spectra obtained from the simulated monofractal (red) and Gaussian (green) series, and for the simulated multifractal series (blue). When the data are multifractal, a fully formed inverted-U shape curve is obtained. However, when the data are monofractal a curve with very small width is obtained, its maximum value of 1 occurring at the value of the single Hurst exponent on the horizontal axis of the graph. 


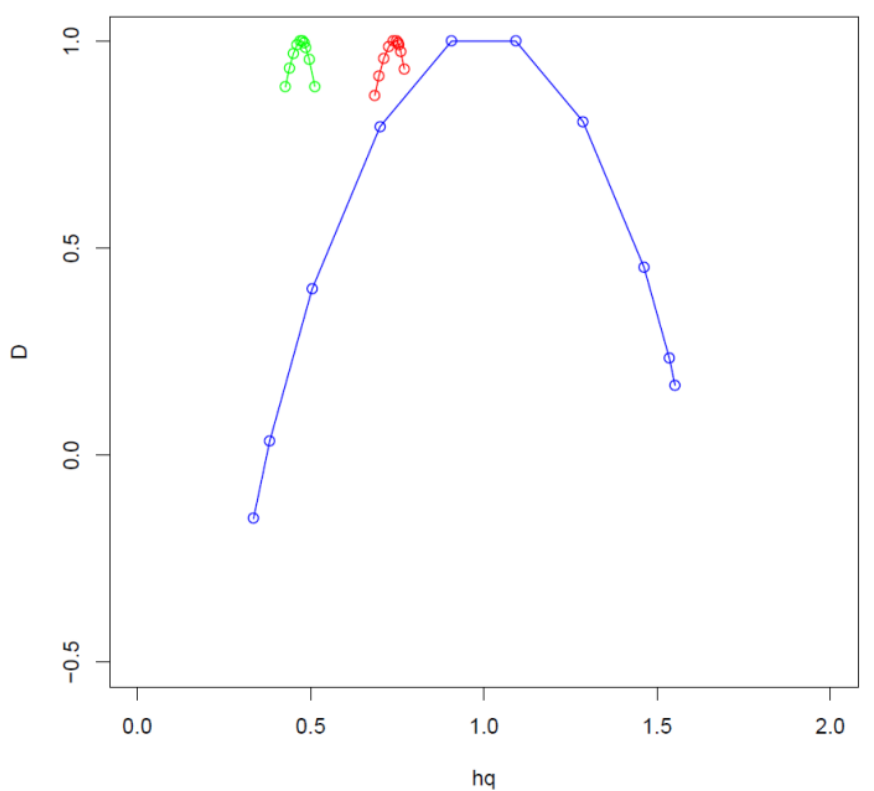

Figure 2. The multifractal spectra for Gaussian noise (green), and simulated monofractal (red) and multifractal (blue) series obtained by applying Multifractal Detrended Fluctuation Analysis to data from Ihlen (2012).

\section{Representation of a Multifractal Process in terms of a Gaussian Multiplicative}

\section{Cascade}

One possible representation of a multifractal process is in terms of a multiplicative cascade as illustrated in Figure A1 in the Appendix. The cascade operates on increasingly more precise time scales starting by dividing the full temporal interval, $T_{0}$, by two at time scale $T_{1}$ and continuing this division by two at each successive time scale, $T_{2}, T_{3}, \ldots, T_{k}$. The flow of information from one time scale to the next is determined by the same probability density function, $p(x)$. Eventually, the hierarchical cascade process can produce the type of activity represented for example during Week 14 by the patient diagnosed with bipolar disorder, or the sequence of heart rates recorded by another patient diagnosed with bipolar disorder. This time series is shown at the bottom of Figure A1 in the Appendix.

The challenge is to determine $p(x)$ based on the form of the multifractal spectrum. For convenience, we will assume that $p(x)$ is the Gaussian pdf with mean, $\mu$, and variance, $\sigma^{2}$, 


$$
p(x)=\frac{1}{\sqrt{2 \pi \sigma^{2}}} \exp \left[-\frac{(x-\mu)^{2}}{2 \sigma^{2}}\right]
$$

Calvert, Fisher and Mandelbrot (1997) have shown that the multifractal spectrum for a Gaussian pdf is given by

$$
f(\alpha)=1-\frac{1}{2 \log (2)}\left(\frac{\alpha-\mu}{\sigma}\right)^{2}
$$

This theoretical multifractal spectrum attains its maximum value of 1 when $\alpha=\mu$ and its width at $f(\alpha)=0$ is given by $0.2355 \sigma$. This means that in principle at least, estimates of the mean and variance of the presumed Gaussian pdf can be obtained by mere inspection of the empirical multifractal spectrum. Fitting Eq. (2) to the multifractal spectrum obviates the need to estimate its peak location and width, two commonly used quantifiers of an empirical multifractal spectrum. It provides a simple test of the Gaussian multiplicative cascade process model, with its implications for a novel theoretical investigation of the temporal precursors of human activity and heart rate generation.

As Calvert et al. have shown, and as is explained in more detail in the Appendix, the probability associated with each branch of a multiplicative cascade process can be constructed by applying a logarithmic transform to the product of random variables that are associated with that branch. This results in a random variable equal to the sum of the logarithm of the Gaussian random variable, the latter being represented by a lognormal pdf with parameters $\mu$ and $\sigma$. The continuous Shannon entropy, $E(\mu, \sigma)$, for this lognormal pdf is given by $E(\mu, \sigma)=\log \left(2 \pi \sigma^{2}\right)+\mu+0.5$ (see Appendix for derivation). In the analyses of activity data, $E(\mu, \sigma)$ is computed for each week of data, using the $\mu$ and $\sigma$ parameter estimates computed from the best-fitting Gaussian multifractal spectrum. With a greater amount of heart rate data, $E(\mu, \sigma)$ can be estimated in a similar way every day.

Although no previous studies have applied multifractal analysis to activity data acquired from a subject susceptible to manic episodes, Ohashi, Yamamoto and Teicher 
(2014) have shown how actigraphy measures can be analysed by the multifractal Hurst function, the precursor of the multifractal spectrum, to assess the effectiveness of light therapy for people suffering from Seasonal Affective Disorder. For those who responded to the light therapy, the Hurst Exponent decreased significantly suggesting a multifractal process. However, the effect was quite small. Application of multifractal analyses to EEG data has shown that the peak location of the multifractal spectrum decreases as the level of consciousness increases from deep sleep to the awake state (Torick \& Mandelkern, 2013). According to the Gaussian multiplicative cascade model, this result implies that the mean of the Gaussian process driving the multiplicative cascade may be inversely related to a person's level of consciousness.

There are different effects of pathology on the width of the multifractal spectrum, depending on the application. Whereas Dutta, Ghosh, Samanta and Dey (2014) have shown that spectrum width measured using extracranial EEG data is greater for those susceptible to epileptic seizures than for healthy individuals, studies using physiological measures, such as heart rate, have shown that people with pathological conditions, such as congestive heart failure, produce multifractal spectra with considerably reduced width when compared with healthy individuals (Ivanov et al., 1999). It is predicted that as a manic episode approaches for a person diagnosed with Bipolar I Disorder, a reduction in multifractal spectrum width will occur, possibly resulting from a decrease in the variance of a Gaussian multiplicative cascade that generates the activity series. This may reflect the added constraints on psychological function when a relapse is imminent. Similar reductions in multifractal spectrum width are expected when people diagnosed with bipolar disorder become quite unwell or are about to experience a self-harm incident. 


\section{Testing for the Presence of Nonlinearity in Activity Time Series}

To detect nonlinearity in activity and heart rate data, surrogate comparison series are obtained by computing the power and phase spectra of the original data series, randomly permuting the phase spectrum components, and using the inverse Fourier transform to produce a new time series. This time series has the same statistical properties as the original time series in terms of its mean, variance, pdf and autocorrelation function, but without dynamic nonlinearity (Schreiber \& Schmitz, 2000).

For each transformed activity or heart rate time series, 30 surrogate comparison series were generated using the TISEAN surrogates routine (Hegger, Kantz \& Schreiber, 1999), available in the nonlinearTseries package for the R Statistical Software (R Core Team, 2021). Evidence for nonlinearity based on the multifractal spectrum was provided by comparing the spectrum produced by the original activity data with the comparison multifractal spectrum computed using the surrogate data (Heath, 2000).

\section{Using A Gaussian Multiplicative Process to Detect Bipolar Risk and}

\section{Presence}

Using data obtained from Professor Greg Murray of Swinburne University, Australia (Indic et al., 2011), analyses were performed on activity data recorded by an ActiGraph over a seven-day period. Participants provided informed consent based on an approved protocol by the Research Ethics Committee at Swinburne University, Australia.

In Experiment 1, activity data were obtained from two groups containing 15 participants each, these being people diagnosed with bipolar disorder and their matched controls, respectively. In Experiment 2, two groups were selected from those people who according to a written questionnaire had the highest and lowest risk of bipolar disorder. For each seven-day activity record, the data were accumulated over five successive observations 
to remove autocorrelation, so that each number represents total activity recorded in each tenminute period.
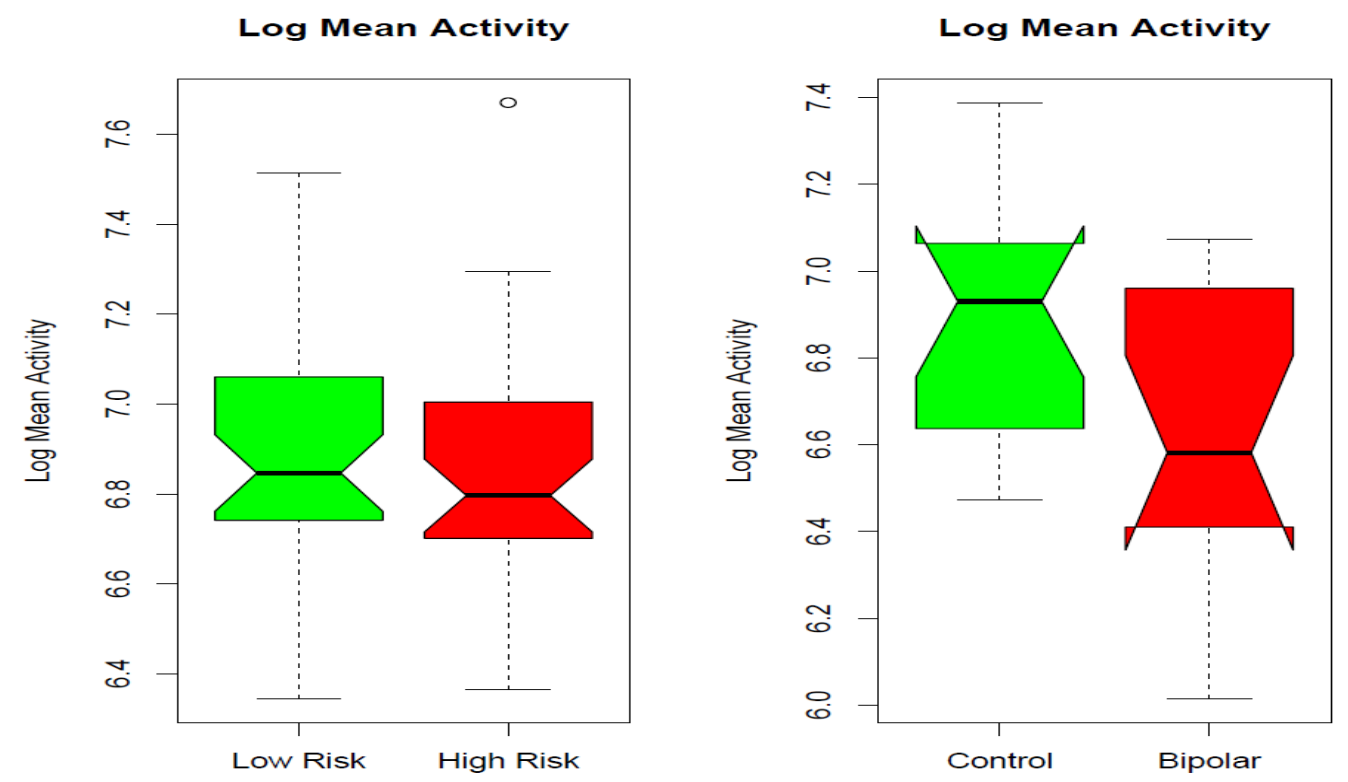

Figure 3. Boxplots of the Log Mean Activity for people with a high and low risk for bipolar disorder (left graph, Experiment 1) and for people diagnosed with bipolar disorder and their matched controls (right graph, Experiment 2)

As shown by the boxplots in Figure 3, there was no difference in log mean activity for the Low and High Risk groups, but there was significantly less log mean activity for the Bipolar group when compared with the Control group, $t(26)=2.09, p=.046$ (Welch Correction).

Activity values, pooled over 10 mins and first differenced to remove trend were analysed using a multiplicative cascade model driven by a homogeneous Gaussian process. The multifractal spectrum was estimated using MFDFA for the data series and 30 surrogate controls. Figure 4 shows multifractal spectra estimated from a week of activity data for selected participants in Experiment 1 who were diagnosed with bipolar disorder and their Matched Controls. The prediction of a multiplicative cascade driven by a homogeneous Gaussian stochastic process is shown by the thick black line. The green lines show the multifractal spectra estimated for 30 surrogate series that have the same linear properties as the activity series but without the nonlinearity. The legend shows estimates of the mean and 
standard deviation of the Gaussian process, LN(mean, standard deviation), the MSE for each model fit and the Entropy for the data and mean surrogate series. Using the Entropy values estimated for the data and surrogate series, Relative Entropy was defined as the difference between Entropy estimated from the data series and the mean Entropy estimated from the surrogate series. When this difference was divided by the standard deviation of the Entropy estimates from the surrogate series, the resulting ratio was defined as zEntropy.

An independent samples t-test conducted on the 12 Bipolar data samples and 10 Control data samples for which a Gaussian model provided a good fit did not detect any significant differences in the Gaussian parameters, Entropy, Relative Entropy or zEntropy. Perhaps this technology was not sufficiently sensitive to detect any change in activity over a period as short as one week, even though some of the participants were diagnosed with bipolar disorder, and there were reasonably accurate fits of the Gaussian model to $73 \%$ of the multifractal spectra estimated from the activity data.

Figure 5 shows multifractal spectra estimated from a week of activity data from selected participants in Experiment 2 who obtained scores indicating a High Risk and Low Risk for bipolar disorder. The prediction of a multiplicative cascade driven by a homogeneous Gaussian stochastic process is shown by the thick black line. The green lines show the multifractal spectra estimated for 30 surrogate series.

An independent samples t-test conducted on the 30 High Risk and 29 Low Risk data samples for which a Gaussian model provided a good fit did not detect any significant differences in the Gaussian parameters, Entropy, Relative Entropy or zEntropy. It is highly likely that this technology was not sufficiently sensitive to detect any change in activity over a period as short of one week, even though some of the participants were considered at High Risk for bipolar disorder. This result occurred despite the reasonably accurate fits of the Gaussian model to $84 \%$ of the multifractal spectra estimated from the activity data. 
(a)

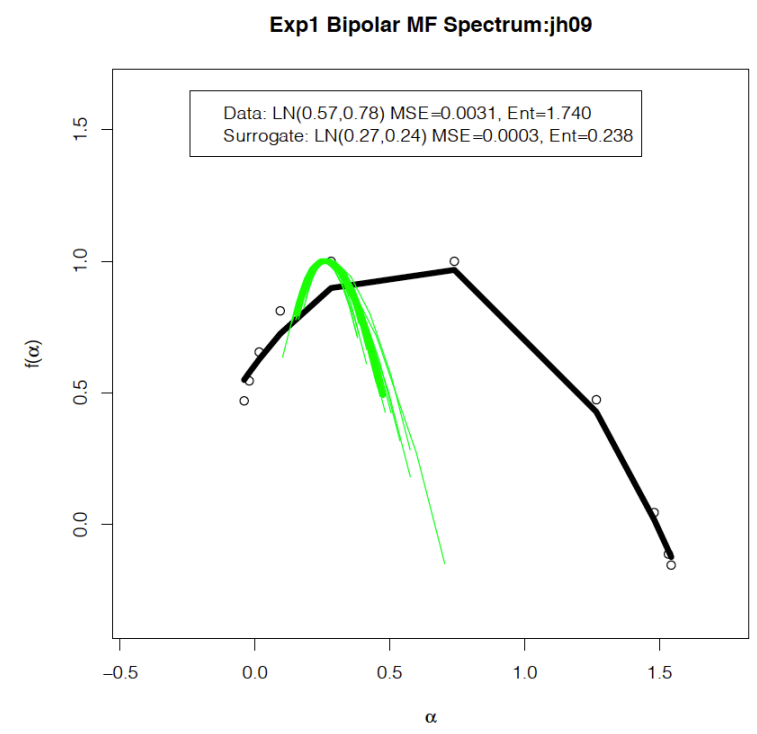

(c)

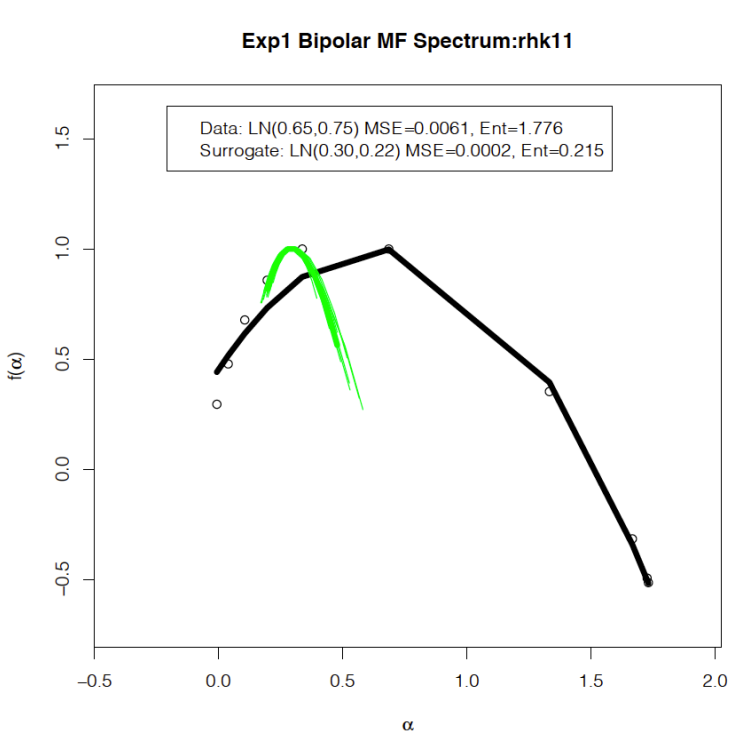

(b)

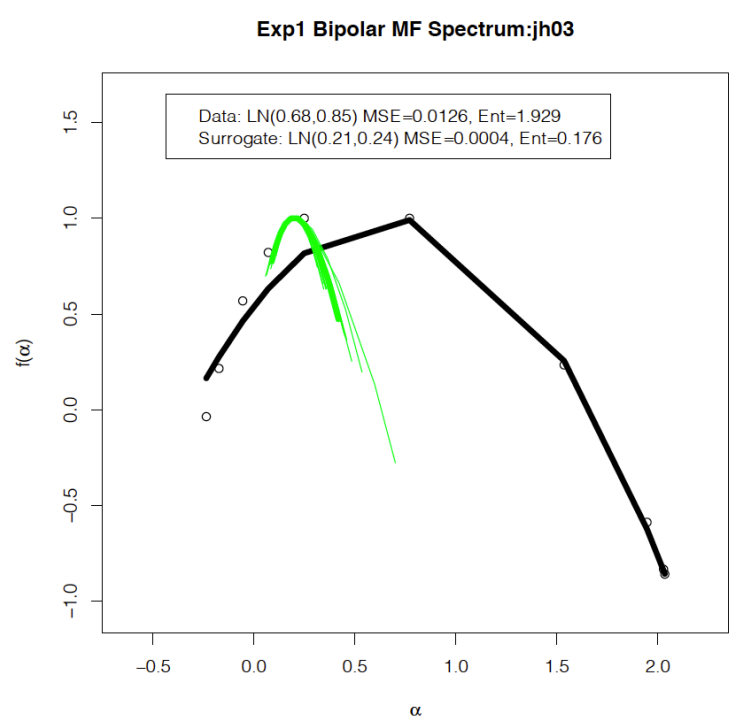

(d)

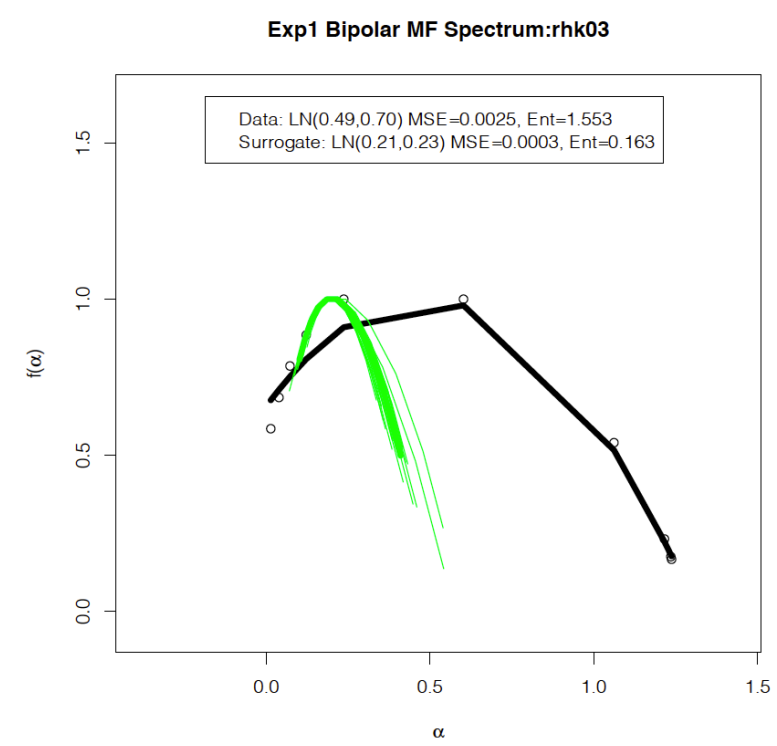


(e)

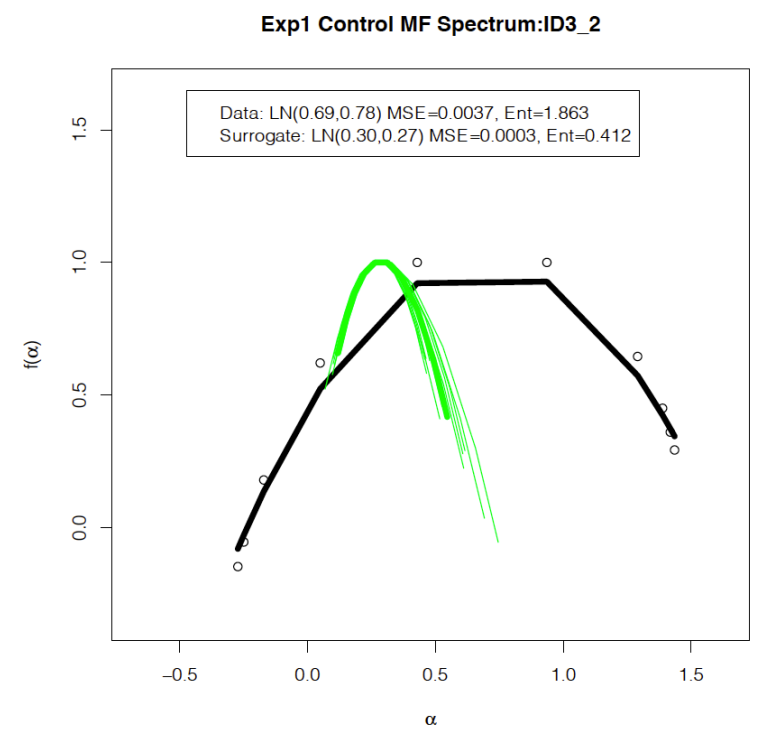

$(\mathrm{g})$

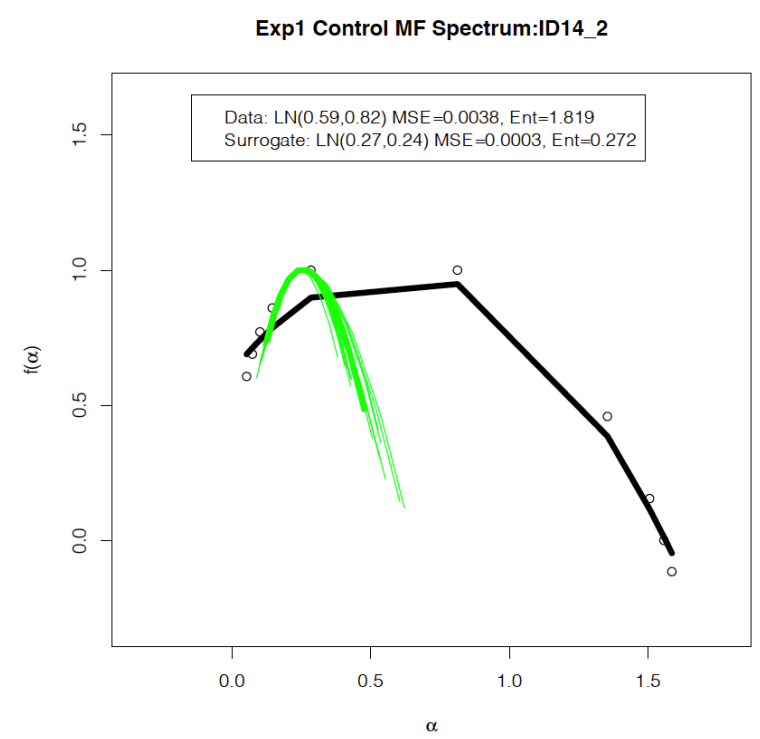

(f)

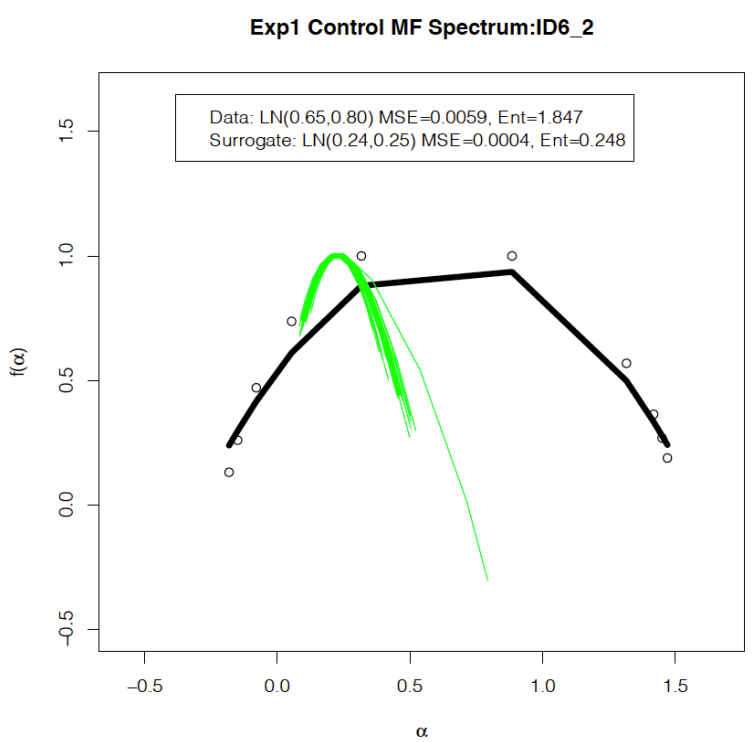

(h)

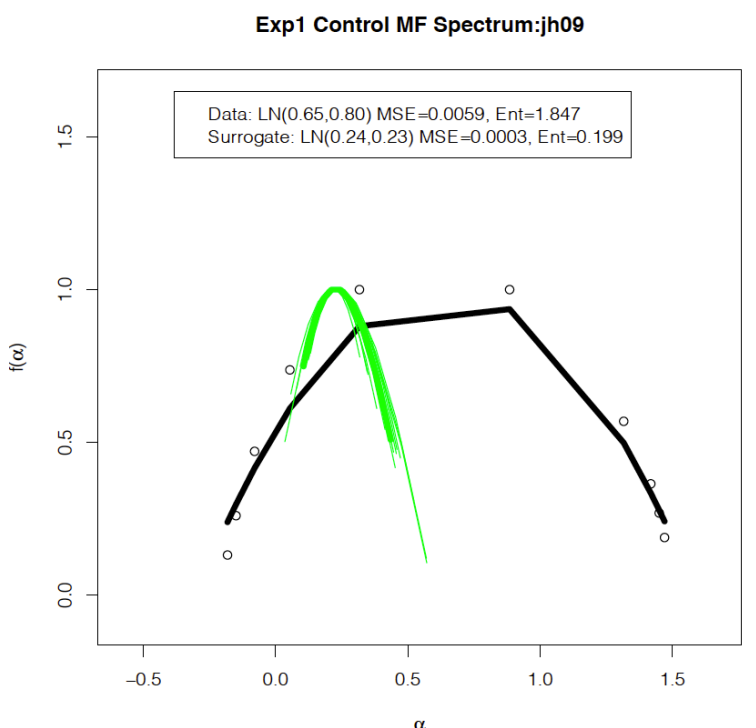

Figure 4. Multifractal spectra (black unfilled dots) estimated from a week of activity data from selected participants in Experiment 1 who were diagnosed with bipolar disorder $(\mathrm{a}-\mathrm{d})$ and their Matched Controls $(\mathrm{e}-\mathrm{h})$. The prediction of a multiplicative cascade driven by a homogeneous Gaussian stochastic process is shown by the thick black line. The green lines show the multifractal spectra estimated for 30 surrogate series that have the same linear properties as the activity series but without the nonlinearity. The legend shows estimates of the mean and standard deviation of the Gaussian process, LN(mean, standard deviation), the MSE for each model fit and the Entropy estimate for the data and mean surrogate series. 
(a)

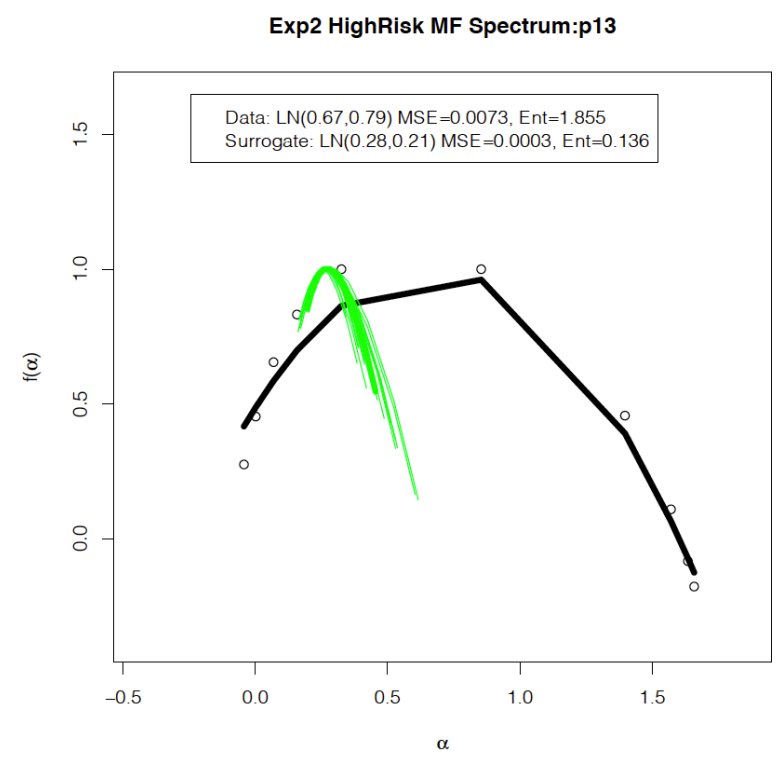

(c)

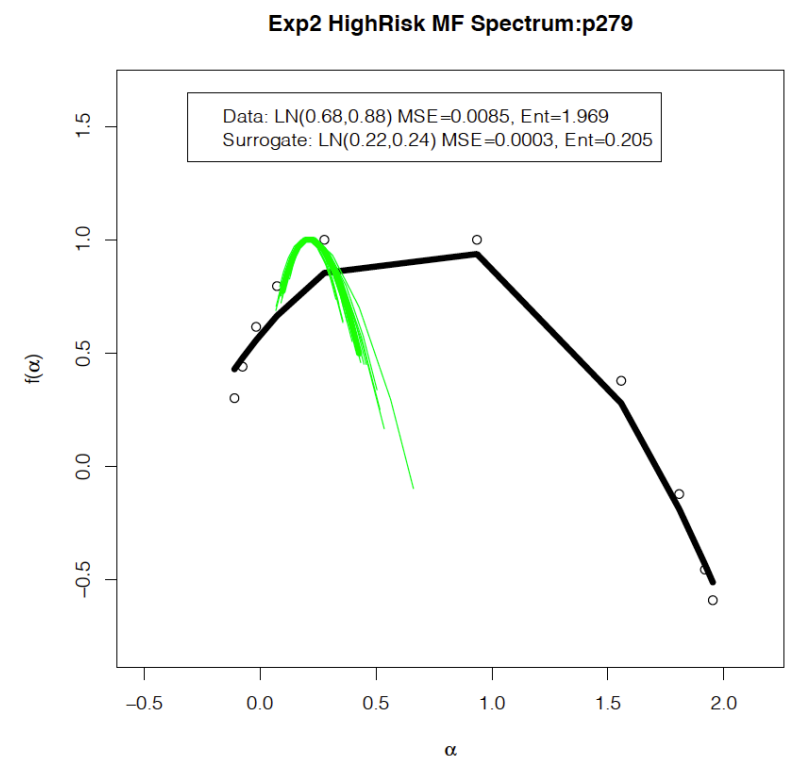

(b)

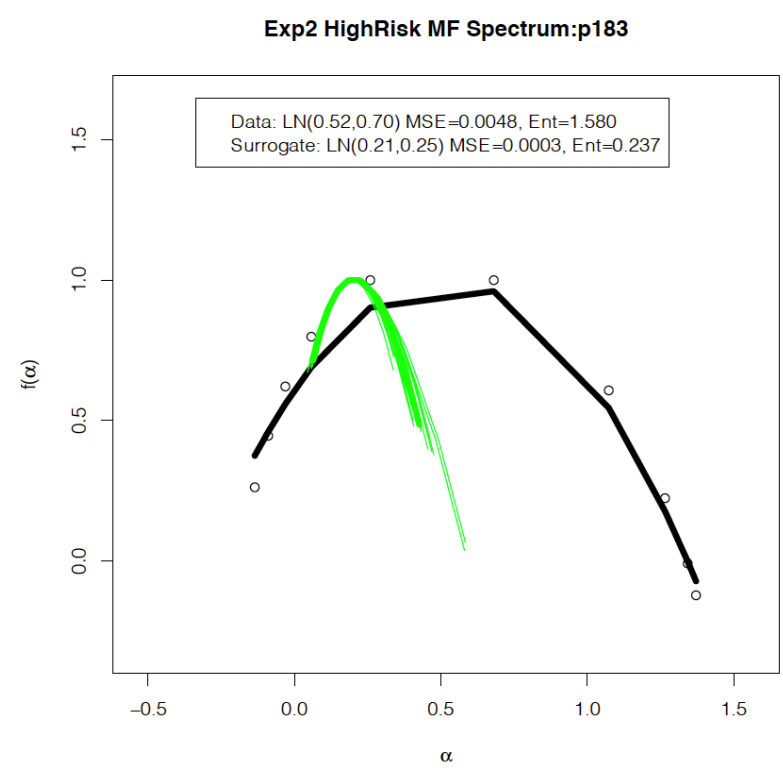

(d)

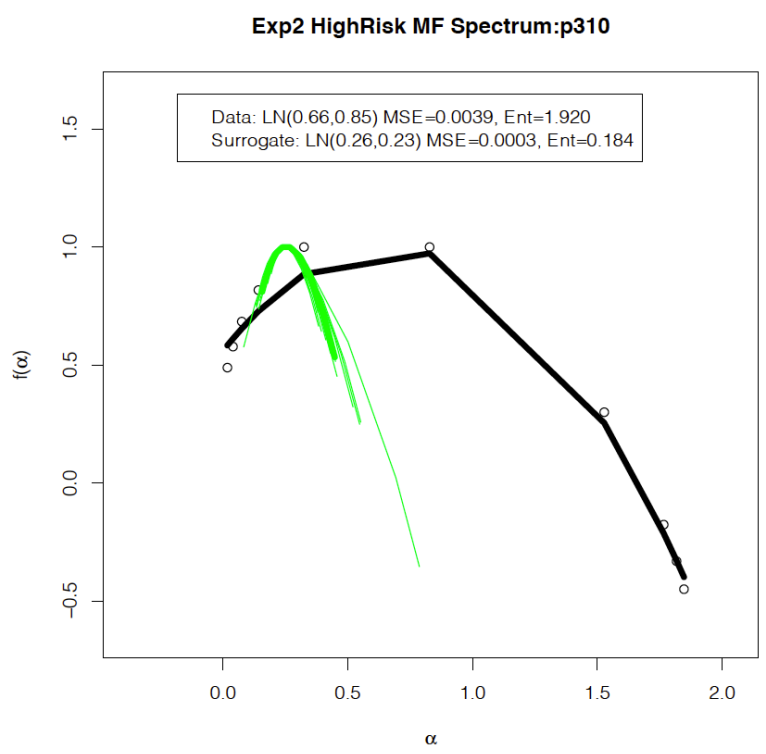


(e)

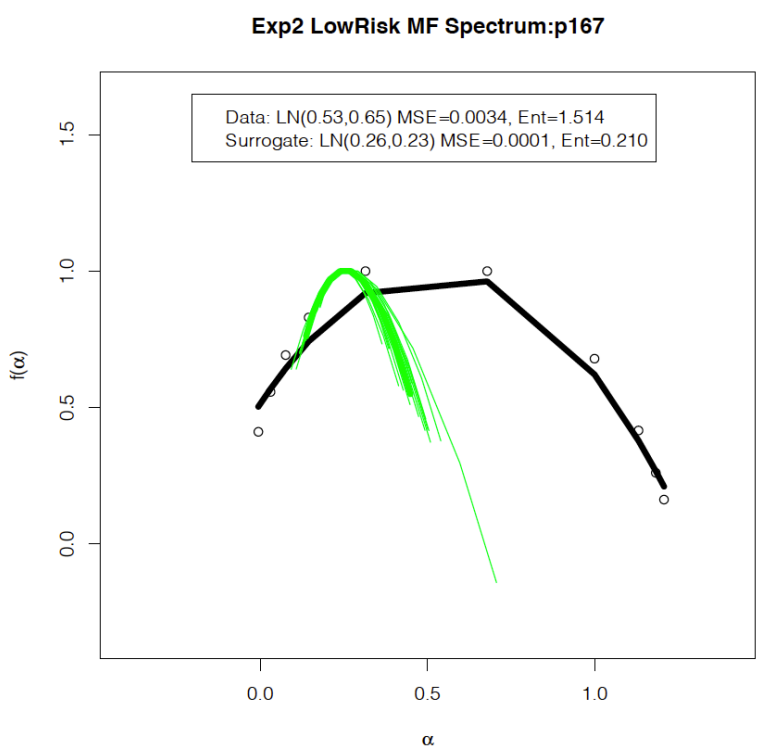

$(\mathrm{g})$

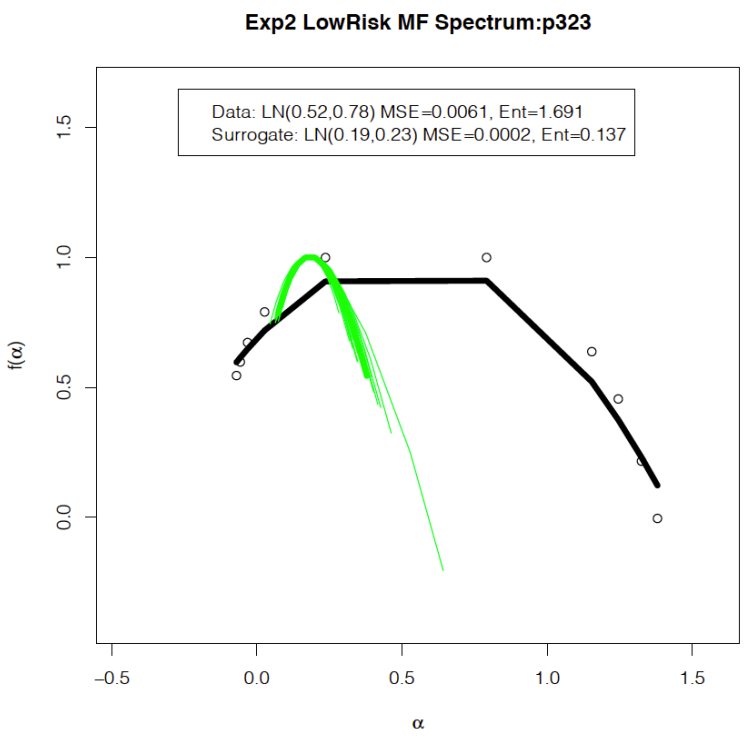

(f)

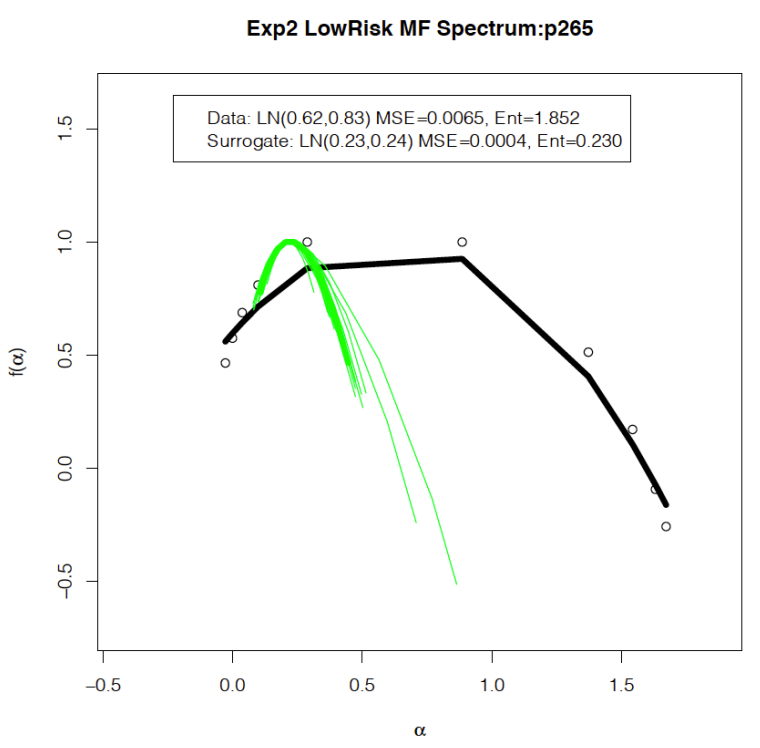

(h)

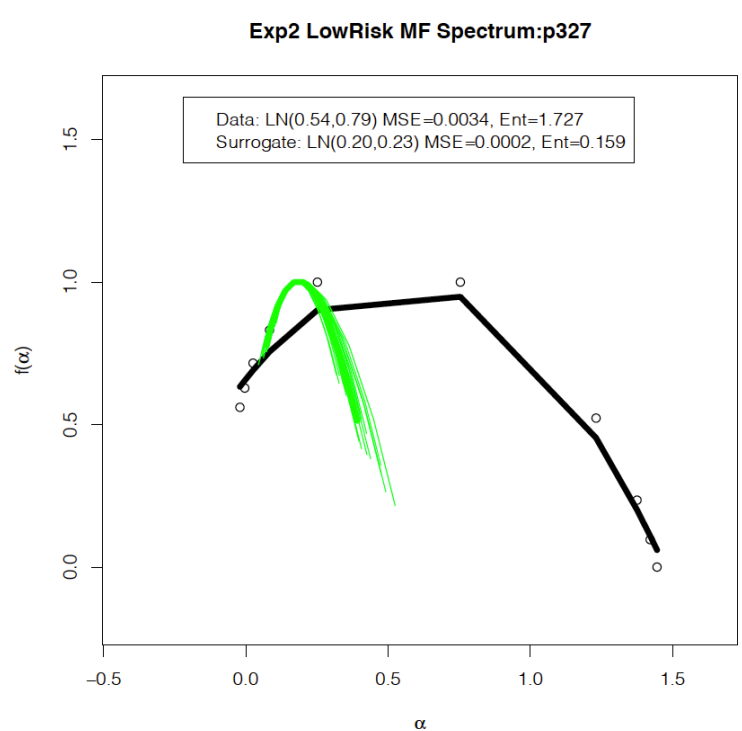

Figure 5. Multifractal spectra (black unfilled dots) estimated from a week of activity data from selected participants in Experiment 2 who obtained scores indicating a High Risk (a-d) and Low Risk (e-h) for bipolar disorder. The prediction of a multiplicative cascade driven by a homogeneous Gaussian stochastic process is shown by the thick black line. The green lines show the multifractal spectra estimated for 30 surrogate series that have the same linear properties as the activity series but without the nonlinearity. The legend shows estimates of the mean and standard deviation of the Gaussian process, LN(mean, standard deviation), the MSE for each model fit and the Entropy for the data and mean surrogate series.

\section{Findings from a Multifractal Analysis of Activity Data from a Single Case}

A multifractal analysis of activity data for the participant who was eventually hospitalised with an manic episode used data accumulated over 10 min periods (1008 observations per 
week). The following example analyses are for Week 9, when the young man diagnosed with Bipolar I disorder appeared to be in a relatively well or euthymic phase, and for Week 14, when the manic episode requiring hospitalisation was imminent.

\section{Generalized Hurst Exponent}

The generalized Hurst exponent functions shown in Figure 6 were monotonic decreasing functions of $q$ suggesting that the transformed activity data were multifractal. The functions for Weeks 9 and 14 estimated using the data (blue lines) were significantly different from those estimated using the surrogates (red lines) when $q<1$ for Week 9 and when $q<-1$ for Week 14. In Week 14, there was an inversion in the order of these functions for negative values of $q$.

The green curves in Figure 6 show the generalised Hurst functions estimated from a normal distribution with mean and variance equal to those of the transformed activity data. The 95\% confidence intervals are shown for each point. For weeks 9 and 14, the data Hurst exponent function deviated significantly from one estimated from the normal distributions showing that the data were not Gaussian. If a monofractal test such as DFA had been applied to these activity data, there would have been no evidence that the activity series were nonlinear and monofractal as the values of the Hurst exponent for the data $(0.18)$ and surrogate series (0.23 are quite close when compared with the large difference in Hurst functions at Weeks 9 and 14. A multifractal analysis is required to obtain any useful information about nonlinear processes that might be responsible for generating activity data in humans, as is shown by the large differences between the blue curves in Figure 6 and those generated by surrogate (red) and normally distributed control (green) series. 

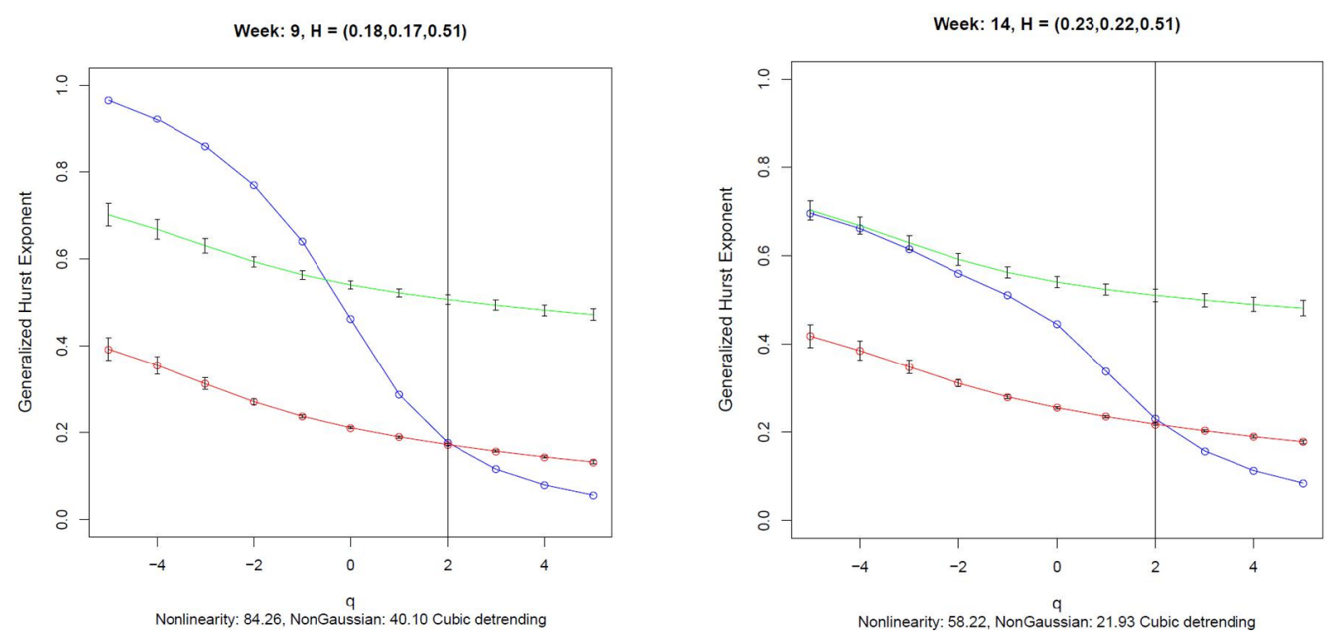

Figure 6. Generalized Hurst Exponent functions for Weeks 9 and 14 estimated using the MFDFA algorithm with cubic detrending. The function for the transformed data (blue) indicates possible nonlinearity when there is no overlap with the $95 \%$ confidence intervals for the function computed using the surrogates (red) and the function generated by a normally distributed random variable with the same mean and variance as the transformed activity data (green). The Nonlinearity Index is the mean difference between the data curve and the surrogate curve in standard normal distribution units. The NonGaussian Index is the mean difference between the data curve and the curve generated from the normally distributed data in standard distribution units. The numbers in brackets are the Detrended Fluctuation Analysis exponents for the data series, surrogate series and normally distributed control series respectively, estimated when $q=2$, as shown by the vertical line.
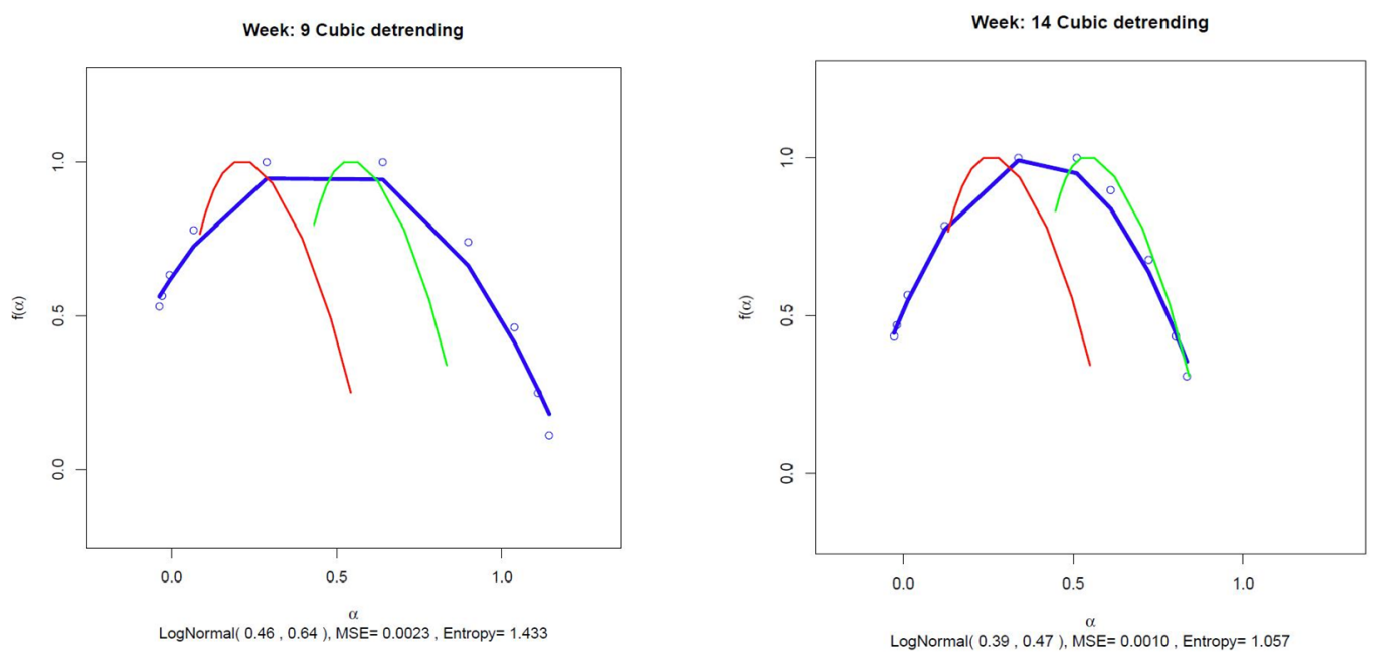

Figure 7. Multifractal spectra for Weeks 9 and 14 estimated using cubic detrending. The blue line shows the best-fitting multifractal spectrum estimated using a multiplicative cascade process driven by a Gaussian pdf. The data points are shown by the small blue circles. The mean multifractal spectra estimated from the surrogate series and the normally distributed control series are shown by the red and green curves, respectively. The lognormal parameter estimates for $\mu$ and $\sigma$ are shown in brackets at the bottom of each graph together with an estimate of the continuous Shannon Entropy for the corresponding lognormal distribution. The MSE for the fit of the lognormal spectrum is also shown.

\section{Multifractal Spectra}

Figure 7 shows the fit of the Gaussian multiplicative cascade model to the multifractal spectra estimated for Weeks 9 and 14. The fit of the model shown by the blue line was reasonably 
good, the peak location of the estimated multifractal spectrum being close to the mean of the lognormal distribution in each case ( 0.46 for Week 9 and 0.39 for Week 14$)$. The width of the multifractal spectrum was positively related to the standard deviation of the Gaussian, being 0.64 in Week 9 and decreasing to 0.47 as the manic episode became established during Week 14. The imminent relapse into a manic state is indicated by the considerable decrease in the width of the multifractal spectra (blue curves) between Week 9 and Week 14, accompanied by decreases in the Gaussian mean $(0.46 \longrightarrow 0.39)$ and Entropy $(1.433 \longrightarrow 1.057)$.

\section{Estimating Change Points in a Time Series}

A commonly employed statistical technique for detecting change points in a time series estimates future values of the series based on a small number of previous values. If the prediction error becomes too large, a change in the parameters governing the time series will have occurred. The method used for this analysis, a semiparametric change detection procedure, can be accessed from commands available in the sac R package (Guan, 2004, 2009). Although the cumsum. test command in sac using the "epidemic" option can be used to detect two change points, the schapt command was also used to compute a semiparametric empirical likelihood ratio test from which the probability associated with the null hypothesis of no change could be estimated. If this value is sufficiently small, a change has most likely occurred and medical intervention might be recommended. The schapt command can then be applied to the remaining data values to detect a second change in the series, a technique used in the heart rate application described in the next section.

Figure 8 shows the estimates of Entropy for a multiplicative cascade driven by a Gaussian process for each of the 14 weeks during which activity data were collected. The multifractal spectrum could not be computed for Week 6 due to missing data. However, a suitable approximation was obtained by linear interpolation of $\mu$ and $\sigma$ by computing the average of their estimates obtained for Weeks 5 and 7. This approximation provided 
estimates of Entropy for detecting change across all 14 weeks of activity observations. Using the change point detection procedure, significant change was detected at Week $5, p=0.011$, and at Week 12, $p=.085$, suggesting that this patient experienced three stages during the period of activity recording, perhaps a recovery period from a previous relapse, a period of relative euthymia and finally the precursors of the manic episode that led to hospitalisation. Although the $p$ value of 0.085 exceeds the usual critical value of 0.05 for a two-tailed statistical test, in this case we hypothesize a decrease in Entropy, so a one-tailed test is more appropriate. In any case, the costs of missing the start of a manic episode, in some cases loss of life, far exceed those of falsely detecting a relapse, so a larger than usual Type I error probability is appropriate in this clinical application.

As shown in Figure 9, the multifractal spectra changed in form over the 14 weeks of activity data. The peak location and width both reached their maximum values during the euthymic period around Week 9. This was followed by declines in both measures until the manic relapse resulted in hospitalisation and no further data collection. This result is shown more clearly when the smoothed width and peak measures are plotted against each other, the curve revealing a full cycle of bipolar disorder from poor health to wellness around weeks 7 to 9 then the progression towards relapse by week 12 .

\section{Application of the Gaussian Multiplicative Cascade Model to Fitbit Heart Rate Data from People Diagnosed with Bipolar Disorder}

Heart rate data were obtained from 11 participants in a study of people diagnosed with bipolar disorder by Dr Tanya Hanstock from the School of Psychological Science at the University of Newcastle, Australia. The project was approved by the Research Ethics Committee of the University of Newcastle, Australia, and all participants provided their informed consent. All the data file descriptors were encrypted to ensure the participants' privacy. All participants provided data series over periods ranging from 7 to 18 weeks using a Fitbit Charge-2 worn on their non-dominant wrist. 


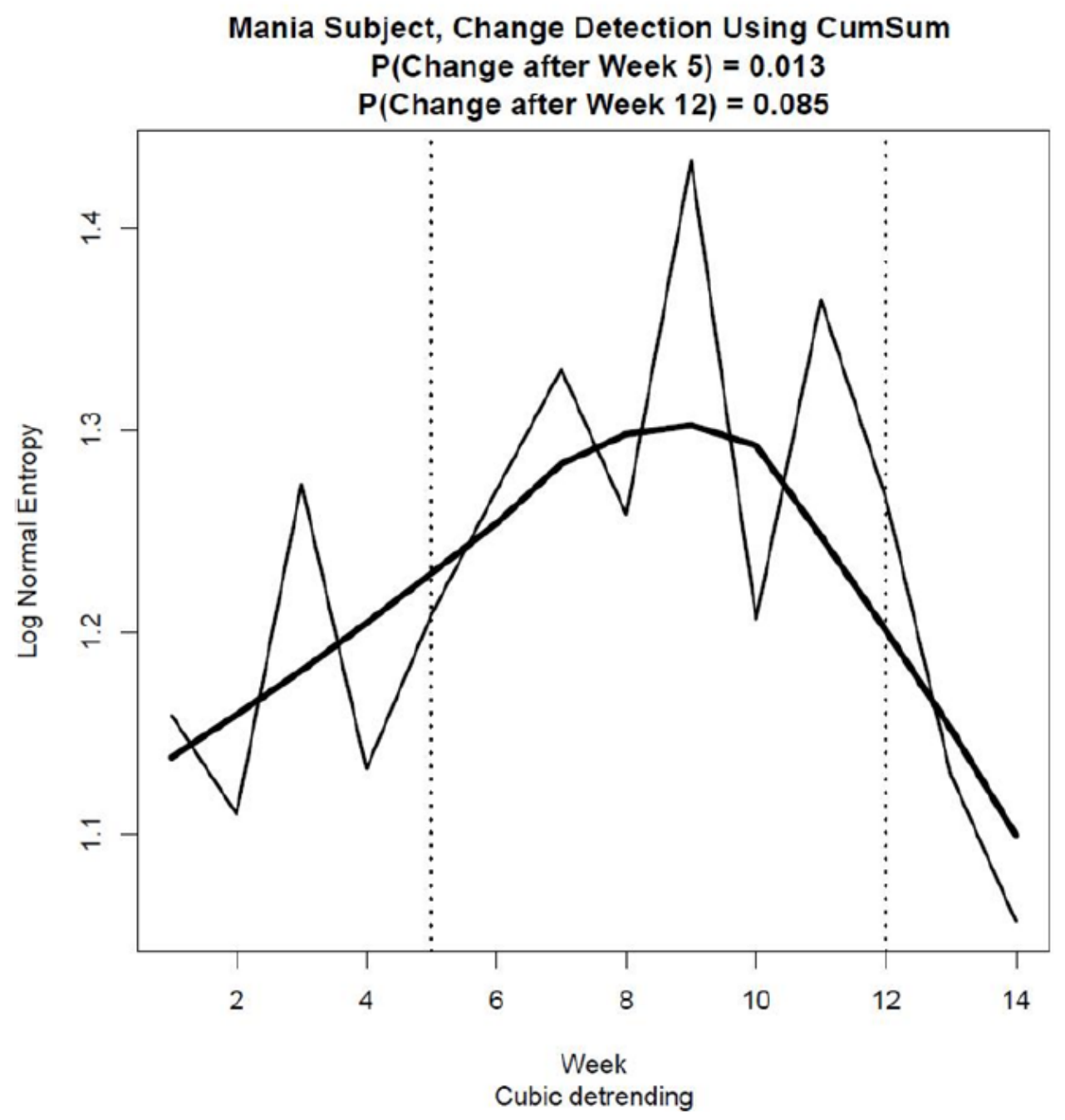

Figure 8. Log Normal Entropy computed using estimates of the mean and standard deviation of a lognormal pdf for transformed activity data collected over a period of 14 weeks prior to the patient's hospitalisation. The vertical lines show a significant increase that occurred at week $5(p=.013)$ and a decrease that occurred at Week $12(p=.085)$. The curve fit to the data points used a Lowess smoothing procedure.

When compared with heart rate recorded by gold standard cardiology devices, the Fitbit heart rate has been shown to be up to 30 bps slower when measured under treadmill conditions, and there may be small constant errors of about 5 bps (Benedetto, Caldato, Bazzan, Greenwood, Pensabene \& Actis, 2018). If these errors are either constant or random, they will simply be sources of noise in the heart rate recordings and not affect the accuracy of the estimated multifractal spectra. 

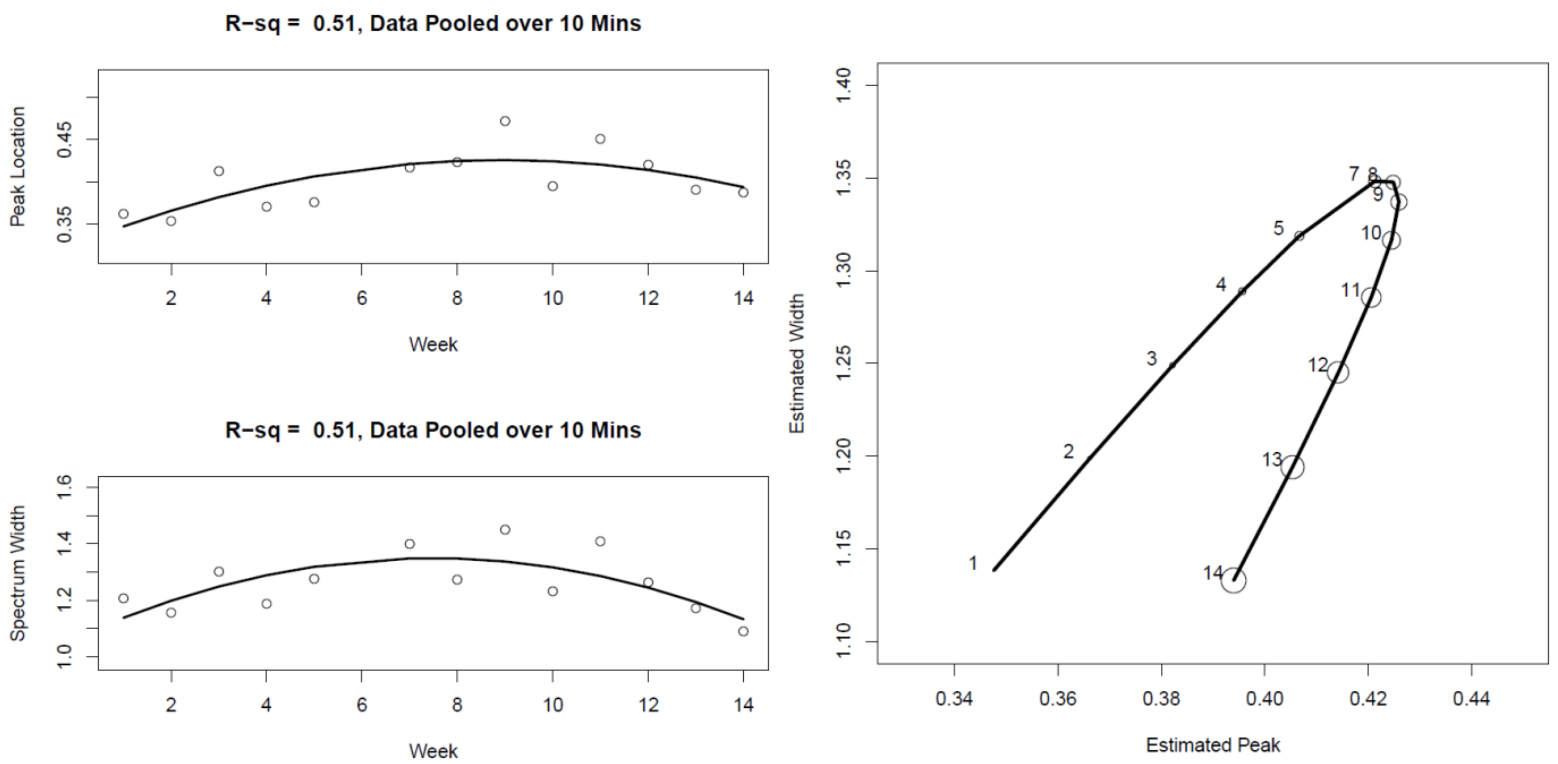

Figure 9. Changes in the multifractal spectrum peak location and width, and the relationship between these features over the 14 weeks of recorded activity data. Week numbers are shown on the right-hand graph.

When the Gaussian Multiplicative Cascade Model (GMCM) was applied to heart rate data pooled over each five minute period and differenced for each of the 11 participants, excellent fits to the data and average surrogate multifractal spectra were obtained. Figure 10 shows examples of a week's data and the corresponding multifractal spectra fits for participants 1 and 4 in their first and last weeks of data recording. Excellent fits of the GMCM were obtained for most of the weekly data for all 11 participants. Relative Entropy, the difference in Entropy estimated from the data multifractal spectrum and from the mean surrogate multifractal spectrum was computed every day for each of the 11 participants. This measure was defined as a Relative Entropy Wellness Index for monitoring change during the observation period that ranged from 7 to 18 weeks. The change detection method was used to detect up to two significant changes during each participant's daily Relative Entropy profile. Relative Entropy Wellness Index profiles are shown for all participants in Figure 11. 


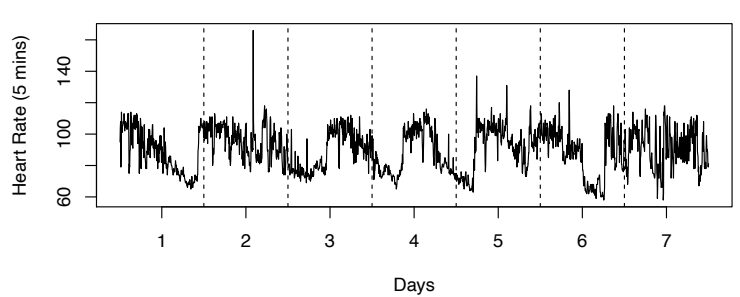

Subject1 Heart Rate (5 mins) Period 1
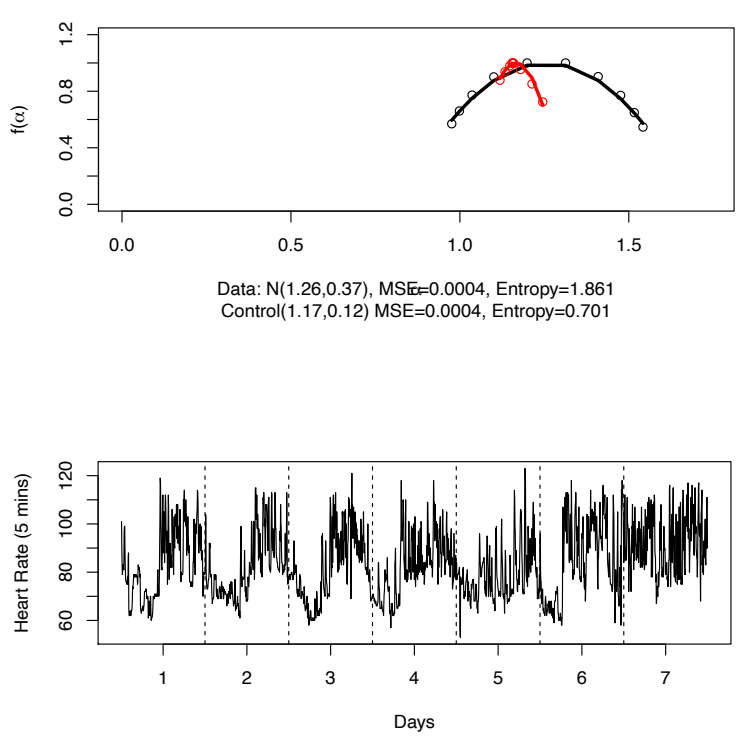

Subject4 Heart Rate (5 mins) Period 1

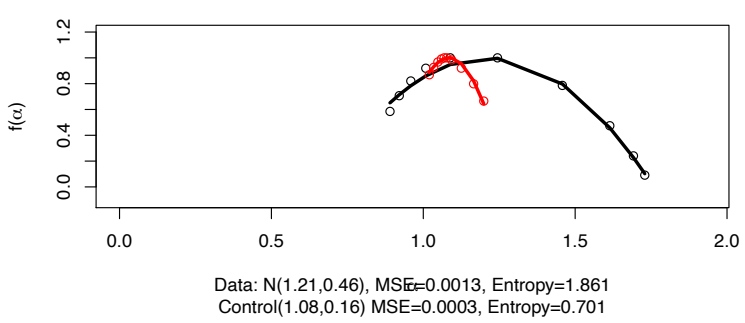

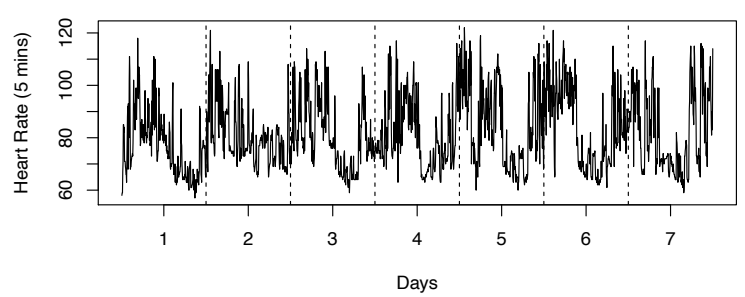

Subject1 Heart Rate (5 mins) Period 10
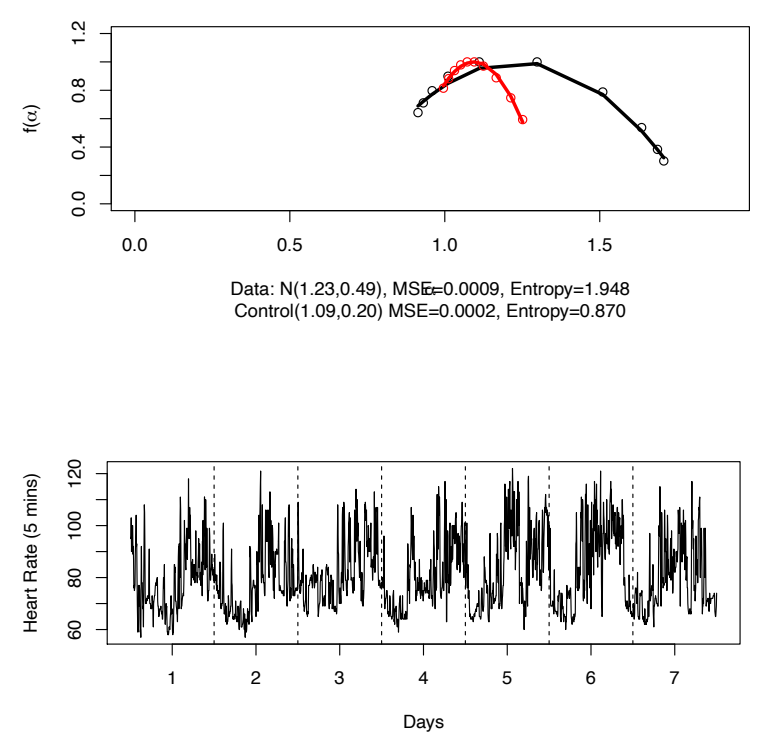

Subject4 Heart Rate (5 mins) Period 9

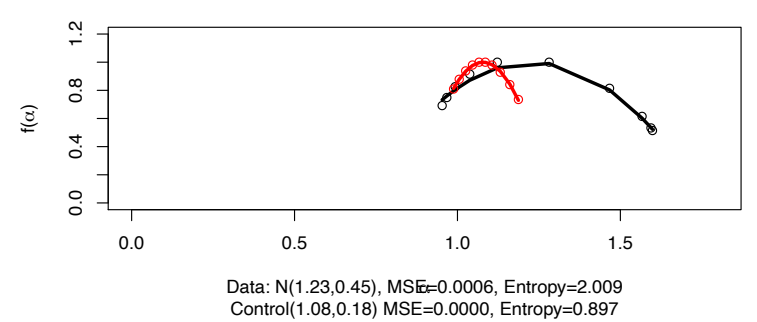

Figure 10. Pooled heart rate recorded over a week for two participants. Data are shown for the first and last week of the recordings. The multifractal spectra are shown by the unfilled black points and the average spectrum from 30 surrogate series is shown by the unfilled red points. Fits of the Gaussian Multiplicative Cascade Model to all these multifractal spectra are also shown by the solid black (data) and red (surrogates) lines. 

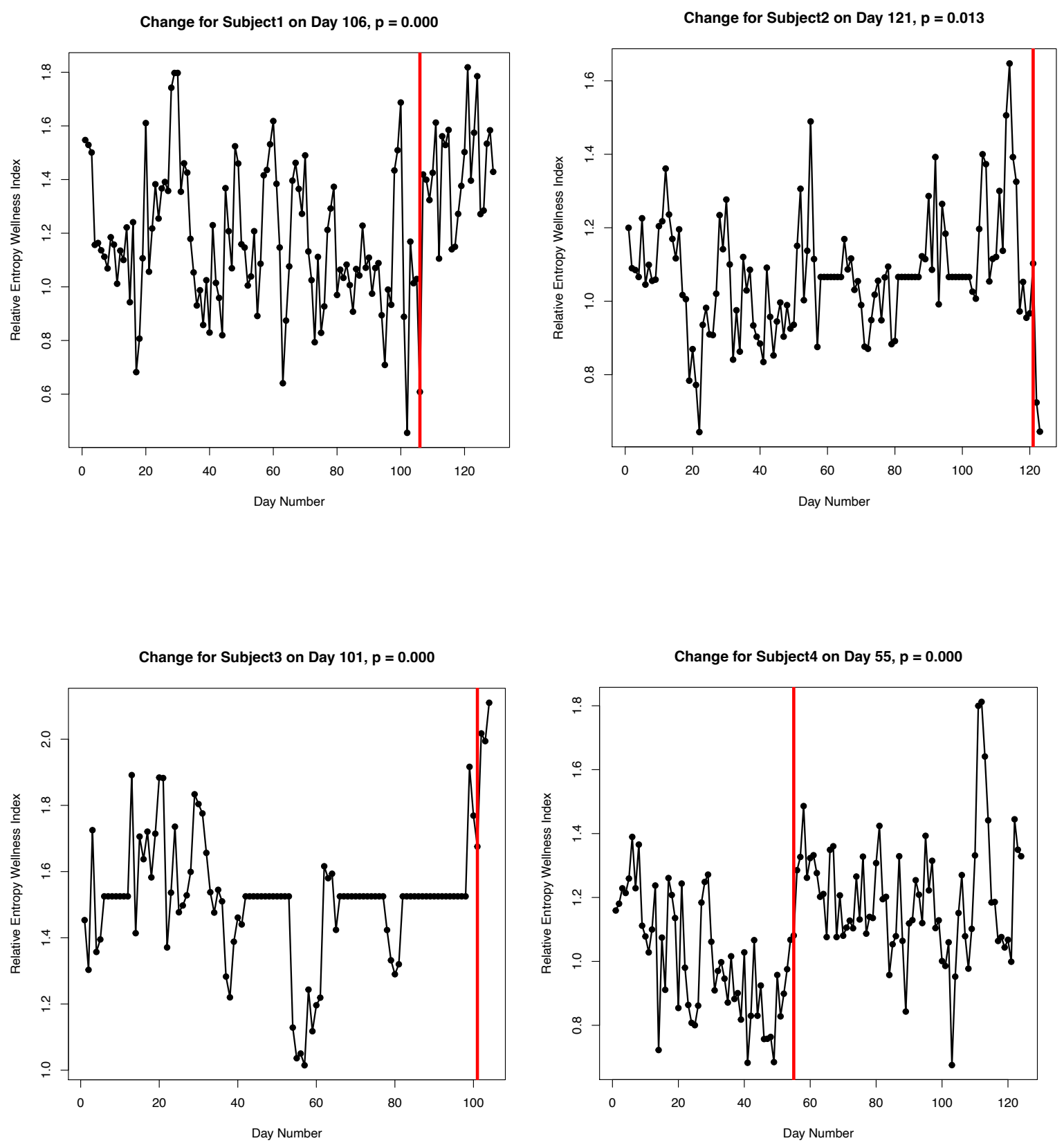

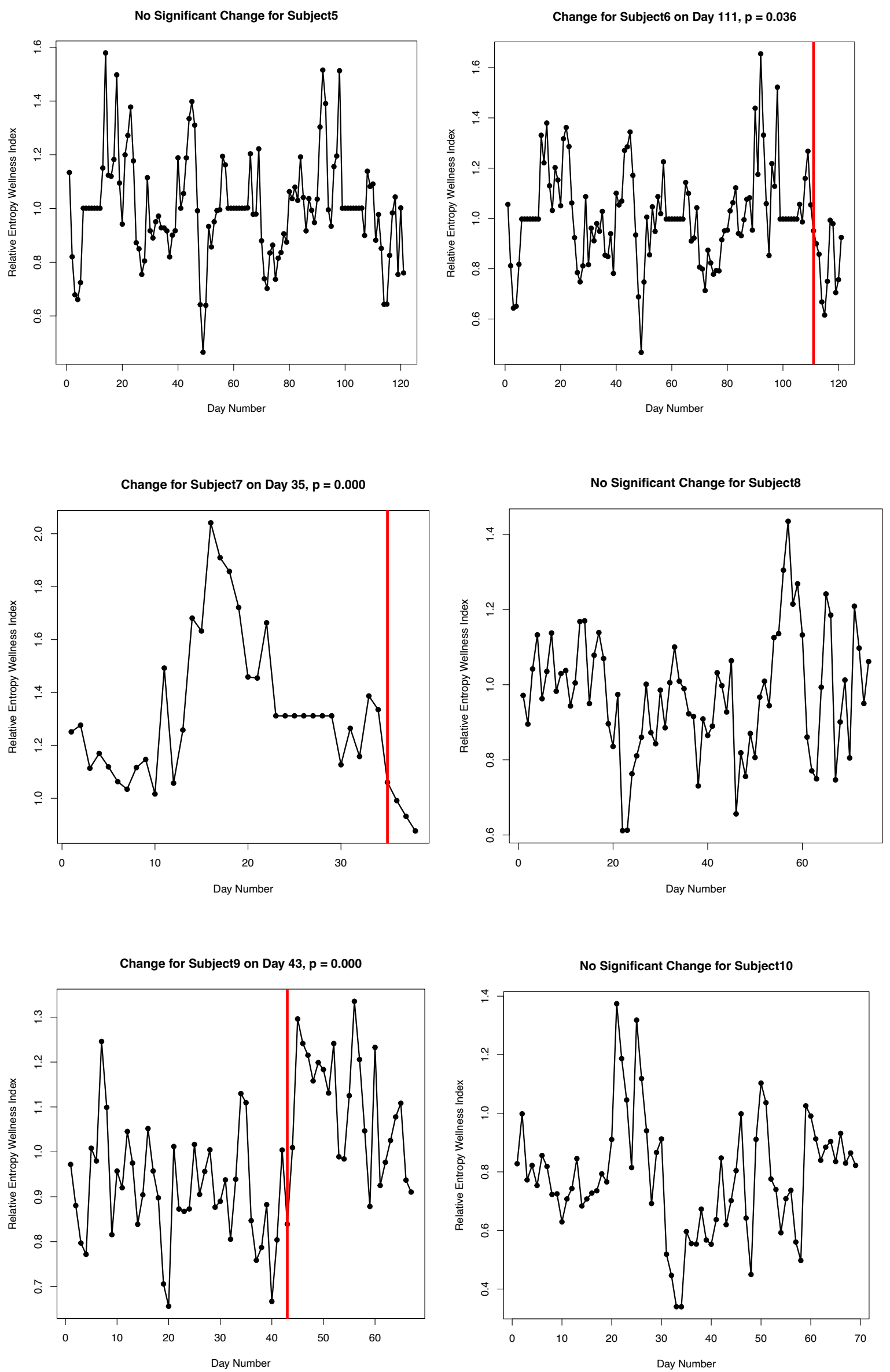


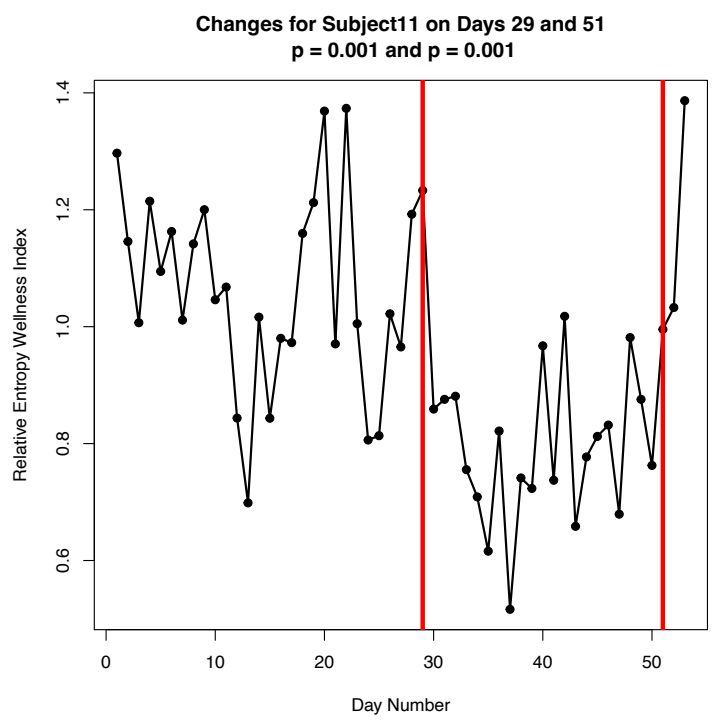

Figure 11. Relative Entropy Wellness Index plots for all 11 participants. Statistically significant changes in Wellness Index are indicated by the vertical red lines. When the MSE for the fit of the Gaussian Multiplicative Cascade Model to the data multifractal spectrum is not less than 0.02 , the points are replotted as the mean of all the Wellness Index values for that participant.

Figure 11 shows the Relative Entropy Wellness Index plots for all 11 participants diagnosed with bipolar disorder. The vertical red lines show days on which a significant change $(p<.05)$ occurred in the Wellness Index. For four participants $(1,3,4$ and 9$)$ there were significant improvements in Wellness, and for three others $(5,8$ and 10) there was no change. Of concern was the reduction in Wellness shown for participants 2, 6, 7 and 11. Participant 2 showed a nonsignificant downturn in Wellness around Day 20 but there was a precipitous decrease detected by Day 121 a few days before this person was rushed to the hospital emergency department after a self-harm incident. Participant 7 has a rapid decline in Wellness detected by Day 35 when they were too unwell to continue their participation in the experiment. Participant 11 was the only one that showed a decrease in Wellness followed by a significant recovery.

\section{Conclusions}

In a proof-of-concept investigation of a single case, activity data acquired from a wrist-worn ActiGraph was used to detect quantitative precursors of a manic episode that resulted in the 
hospitalization of a young man diagnosed with bipolar disorder. Using transformed activity data, the width and peak location of multifractal spectra were computed using Multifractal Detrended Fluctuation Analysis for each of the 14 weeks of recordings. There was a significant decrease in multifractal spectrum peak location and width during the two-week period prior to the onset of the manic episode on the 104th day. This finding was confirmed when an Entropy measure derived from a log Gaussian distribution decreased significantly as the episode approached, a finding consistent with the idea that a decrease in complexity accompanies periods of relapse in a chronic illness. As this analysis is based on a single case study and just one-episode, larger samples will need to be prospectively monitored for manic and depressive relapses to generalize the proposed methodology and permit its widespread usage for automated episode monitoring and prevention.

For the first time, it was shown that activity data recorded over several months for a patient on the verge of a manic episode were clearly multifractal. The multifractal spectra were consistent with those predicted by a multiplicative cascade process driven by a single Gaussian distribution. From this model, a diagnostically useful Entropy value could be obtained using estimates of the Gaussian mean and standard deviation. Application of statistically sound change detection methodology revealed three stages during the observation period. Corroborated by intraday findings for this person (Heath, 2022, February 28), the first stage represented a period of low entropy, higher activity during the day when compared with the evening and a moderate overall level of activity. The second stage, a period of relative wellness, revealed the highest Entropy during a period of greater activity during the day when compared to the evening, as well as a tendency towards greater overall activity. The final stage indicated a period of roughly equal activity throughout the day and night as well as the continuation of the tendency towards increased overall activity as the manic episode became established. This period was associated with a dramatic decrease in Entropy that was almost 
statistically significant using the conventional 0.05 level of significance. Based on research in physical diseases, such a decrease in Entropy would be associated with the onset of deterioration for those diagnosed with a chronic disorder.

When considered in terms a practical decision-making strategy for assessing the risk of a manic episode, the false alarm probability of 0.085 has considerable practical significance. If the algorithms described in this paper were used in a mobile mental health monitoring device, the patient's mental health team would be well-advised to check the patient's current status given that the chance of relapse by Week 12 was reasonably high. Those concerned with the huge costs involved in hospitalisation of patients with a serious mental health problem lasting several weeks if not months would much prefer the minimal cost of preventative intervention by the medical and community mental health team to the immense cost of long-term hospitalisation. If confirmed in future analyses of similar data from bipolar patients, these findings will allow medical intervention to occur well before the highly disrupted sleep that was observed by Bullock, Judd and Murray (2014) about five days before the manic episode occurred. This technological innovation will serve as a valuable contribution towards an improved standard of care for people diagnosed with bipolar disorder, as well as those diagnosed with other serious chronic illnesses.

Evidence from a study of patients diagnosed with bipolar disorder showed that a Wellness Index estimated from the parameters of the Gaussian Multiplicative Cascade Model applied to Fitbit heart rate data could be used to detect statistically significant change in a person's mental health status. Of special interest were warnings of severe illness in one case leading to that person's departure from the experiment, and the precursor of a self-harm incident requiring emergency intervention for another person. In online applications of the technology, it might have been possible to implement a warning via SMS, for example, to expedite medical intervention before the self-harm incident occurred. 
Evidence for nonlinear processes in the activity data were revealed by the significant discrepancies between generalized Hurst functions computed using the transformed data and functions estimated from surrogate series that had the same linear properties as the data but without the nonlinearity. This finding emphasises the advantage of using an objective measure such as activity rather than a subjective measure such as mood ratings (Moore, Little, McSharry, Geddes, \& Goodwin, 2012, 2014; Ortiz, Bradler, Garnham, Slaney \& Alda, 2014). Moreover, we used technology that provides a comprehensive account of the important multiplicative temporal interactions that might determine mood variation.

The change in multifractal spectrum complexity as the manic episode approached has its precursor in findings that EEG multifractal spectrum complexity, as indicated by spectrum width, increased prior to an epileptic seizure (preictal phase) and decreased dramatically as seizure activity increased. In a study that investigated fluctuations in people's pain experiences, multifractal spectrum complexity was less during periods of pain than when no pain was reported (Dick \& Svyatogor, 2012). Perhaps multifractal spectrum complexity is greater during well periods than at times when a person with a dynamic condition, such as bipolar disorder, is experiencing a well phase. In this single test case, the reduced complexity of the multifractal spectrum may indicate that the usual feedback mechanisms that facilitate human-environmental interaction are disrupted as bipolar patients become unstable. This situation might lead to an inability of the brain regulatory systems to properly manage mood (Ihlen \& Vereijken, 2013).

As the above analyses were based on a manic relapse period for one patient, the generalizability of the findings is uncertain. Nevertheless, it is rare to obtain so much continuous activity data over such a long period of time. Unlike the study by Krane-Gartiser, Henriksen, Morken, Vaaler and Fasmer (2014), there was no opportunity to track activity during hospitalization and subsequent recovery due to removal of the wearable device as part 
of the admission procedure. In future studies it would be worthwhile if the wearable device was worn continuously so that the patient's recovery in hospital could also be monitored.

\section{REFERENCES}

American Psychiatric Association. (2013). Diagnostic and statistical manual of mental disorders: DSM-5, 5th ed. Author, Washington, DC.

Ancoli-Israel, S., Cole, R., Alessi, C., Chambers, M., Moorcroft, W., \& Pollak, C.P. (2003). The role of actigraphy in the study of sleep and circadian rhythms. Sleep, 26, 342392.

Benedetto, S., Caldato, C., Bazzan, E., Greenwood, D.C., Pensabene, V., \& Actis, P. (2018). Assessment of the Fitbit Charge 2 for monitoring heart rate. PLoS ONE 13(2): e0192691. https://doi. org/10.1371/journal.pone.0192691

Borsboom, D. (2017). A network theory of mental disorders. World Psychiatry, 16, 5-13.

Borsboom, D., Cramer, A.O.J., Schmittmann, V.D., Epskamp, S., \& Waldorp, L.J. (2011). The small world of psychopathology. PLoS One, 17; 6(11):e27407

Boschloo, L., van Borkulo, C.D., Rhemtulla, M., Keyes, K.M., Borsboom, D., \& Schoevers, R.A. (2015). The Network Structure of Symptoms of the Diagnostic and Statistical Manual of Mental Disorders. PLoS ONE 10 (9): e 0137621. doi:10.1371/journal.pone.013762

Bullock, B., Judd, F.K., \& Murray, G. (2014). Using actigraphy to monitor sleep-wake patterns in bipolar disorder: a case study. Clinical Psychology Forum, 253, 37-41. 
Bullock, B., \& Murray, G. (2014). Reduced amplitude of the 24 hour activity rhythm: A biomarker of vulnerability to bipolar disorder? Clinical Psychological Science, 2, 8696.

Burton, C., Heath, R.A., Weller, D., \& Sharpe, D. (2010). Evidence of reduced complexity in self-report data from patients with medically unexplained symptoms. Nonlinear Dynamics in Psychology and Life Sciences, 14, 15-25.

Cairney, J., Veldhuizen, S., Faulkner, G., Schaffer, A., \& Rodriguez, C. (2009). Bipolar disorder and leisure-time physical activity: results from a national survey of Canadians. Mental Health and Physical Activity, 2, 65-75.

Calvert, L., Fisher, A., \& Mandelbrot, B. (1997). Large deviations and the distribution of price changes. Cowes Foundation Discussion Paper No. 1165. New Haven, CT: Yale University.

Delignières, D., Torre, K., \& Lemoine, L. (2011). Methodological issues in the application of monofractal analyses in psychological and behavioral research. In Guastello, S.J., \& Gregson, R.A.M. (Eds.), Nonlinear dynamical systems analysis for the behavioral sciences using real data. (pp. 559-582) Boca Raton, FL: CRC Press.

Dick, O.E., \& Svyatogor, I.A. (2012). Potentialities of the wavelet and multifractal techniques to evaluate changes in the functional state of the brain. Neurocomputing, $82,207-215$.

Dutta, S., Ghosh, D., Samanta, S., \& Dey, S. (2014). Multifractal parameters as an indication of different physiological and pathological states of the human brain. Physica A, 396, $155-163$.

Finazzi, M.E., Mesquita, M.E., Lopes, J.R., Fu, L.I., Oliveira, M.G., \& Del Porto, J.A. (2010). Motor activity and depression severity in adolescent outpatients. Neuropsychobiology, 61, 33-40. 
Garcia, C.A., \& Sawitzki, G. (2021). Nonlinear time series package for R. https://cran.r-project.org/web/packages/nonlinearTseries/nonlinearTseries.pdf Downloaded on 13 October, 2021.

Goodwin, F.K., \& Jamison, K.R. (2007). Manic-depressive illness: Bipolar disorders and recurrent depression. New York: Oxford University Press.

Goodwin, G.M. (2003). Consensus Group of the British Association for Psychopharmacology. Evidence-based guidelines for treating bipolar disorder: recommendations from the British Association for Psychopharmacology. Journal of Psychopharmacology, 17,149-173.

Gottschalk, A., Bauer, M.S., \& Whybrow, P.C. (1995). Evidence of chaotic mood variation in mood disorder. Archives of General Psychiatry, 52, 947-959.

Grunze, H. (2010). The treatment of mania. In Young, A.H., Ferrier, I.N., \& Michelak, E.E. (Eds.) Practical management of bipolar disorder. (pp. 10-23) Cambridge, UK: Cambridge University Press.

Guan, Z. (2004). A semiparametric changepoint model. Biometrika, 91, 849-862.

Guan, Z. (2005). The sac package. Version 1.0. Downloaded on 30 October 2015 from http://ftp.auckland.ac.nz/software/CRAN/doc/packages/sac.pdf

Heath, R.A. (2000). Nonlinear dynamics. Techniques and applications in psychology. Mahwah, NJ: Lawrence Erlbaum Associates.

Heath, R.A. (2004). Complexity and mental health. In Holt, T. (Ed.), Complexity for clinicians. (pp. 83-94) Oxford, UK: Radcliffe Medical Press.

Heath, R.A. (2022, February 28). Monitoring mood disorder using activity data. PsyArXiv preprint, https://psyarxiv.com/8kbu6 
Heath, R.A., Heiby, E.M., \& Pagano, I.S. (2007). Complex dynamics in depression: An application to long-term, mood-rating time series. In J. Neufeld (Ed.). Advances in clinical cognitive science: Formal modeling and assessment of processes and symptoms. (pp. 263-292) Washington, D.C.: APA Publications.

Hegger, R., Kantz, H., \& Schreiber, T. (1999). Practical implementation of nonlinear time series methods: The TISEAN package. Chaos, 9, 413-435.

Heiby, E.M., Pagano, I.S., Blaine, D.D., Nelson, K., \& Heath, R.A. (2003). Modelling unipolar depression as a chaotic process. Psychological Assessment, 15, 426-434.

Hu, K., Scheer, F.A., Ivanov, P., Buijs, R.M., Shea, S.A. (2007). The suprachiasmatic nucleus functions beyond circadian rhythm generation. Neuroscience, 149, 508-517.

Ihlen, E.A. (2012). Introduction to multifractal detrended fluctuation analysis in Matlab. Frontiers in Physiology, 3, 141.

Ihlen, E.A.F. (2013). Multifractal analyses of response time series: A comparative study. Behavior Research, 45, 928-945.

Ihlen, E.A.F., \& Vereijken, B. (2010). Interaction dominant dynamics in human cognition: beyond 1/fa fluctuations. Journal of Experimental Psychology: General, 139, 436463.

Ihlen E.A., \& Vereijken, B. (2013). Multifractal formalisms of human behavior. Human Movement Science, 32, 633-651.

Indic, P., Salvatore, P., Maggini, C., Ghidini, S., Ferraro, G., Baldessarini, R.J., Murray, G. (2011). Scaling behavior of human locomotor activity amplitude: Association with bipolar disorder. PLoS ONE, 6(5), e20650. doi:10.1371/journal.pone.0020650 
Ivanov, P.C., Amaral, L.A., Goldberger, A.L., Havlin, S., Rosenblum, M.G., Struzik, Z.R., \& Stanley, H.E. (1999). Multifractality in human heartbeat dynamics. Nature, 399, 461465.

Janney, C. A., Fagiolini, A., Swartz, H. A., Jakicic, J. M., Holleman, R. G., \& Richardson, C. R. (2014). Are adults with bipolar disorder active? Objectively measured physical activity and sedentary behavior using accelerometry. Journal of affective disorders, 152-154, 498-504. https://doi.org/10.1016/j.jad.2013.09.009

Kantelhardt, J.W., Zschiegner, S.A., Koscielny-Bunde, E., Bunde, A., Havlin, S., \& Stanley, H.E. (2002). Multifractal detrended fluctuation analysis of nonstationary time series. Physica A: Statistical Mechanics and its Applications, 316, 87-114.

Kelty-Stephen, D.G., Palatinus, K., Saltzman, E., \& Dixon, J.A. (2013). A tutorial on multifractality, cascades, and interactivity for empirical time series in ecological science. Ecological Psychology, 25, 1-62.

Krane-Gartiser, K., Henriksen, T.E.G., Morken, G., Vaaler, A., \& Fasmer, O.B. (2014) Actigraphic assessment of motor activity in acutely admitted inpatients with bipolar disorder. PLoS ONE, 9(2): e89574. doi:10.1371/journal.pone.0089574

Lam, D., Wong, G. (2005). Prodromes, coping strategies and psychological interventions in bipolar disorders. Clinical Psychology Review, 25, 1028-1042.

Mandelbrot, B.B. (1997). The fractal geometry of nature. New York: Freeman.

Merikangas, K.R., Jin, R., He, J.P., Kessler, R.C., Lee, S., Sampson, N.A., et al. (2011). Prevalence and correlates of bipolar spectrum disorder in the world mental health survey initiative. Archives of General Psychiatry, 68, 241-251.

Moore, P.J., Little, M.A., McSharry, P.E., Geddes, J.R., \& Goodwin, G.M. (2012). Forecasting depression in bipolar disorder. IEEE Transactions on Biomedical Engineering, 59, 2801-2807. 
Moore, P.J., Little, M.A., McSharry, P.E., Goodwin, G.M., \& Geddes, J.R. (2014). Mood dynamics in mood disorder. International Journal of Bipolar Disorders, doi:10.1186/s40345-014-0011-z.

Mullen, K. M., Ardia, D., Gil, D., Windover, D., \& Cline, J. (2011). DEoptim: An R package for global optimization by differential evolution. Journal of Statistical Software, 40, $1-26$.

Murray, G. (2010). Circadian and sleep/wake considerations in the practical management of bipolar disorder. In Young, A.H., Ferrier, I.N., \& Michelak, E.E. (Eds.) Practical management of bipolar disorder. (pp. 152-162) Cambridge, UK: Cambridge University Press.

Ohashi, K., Yamamoto, Y., \& Teicher, M.H. (2014). Locomotor microactivities associated with therapeutic responses in patients with seasonal affective disorders. Integrative Medicine International, 1, 151-161.

Ortiz, A., Bradler, K., Garnham, J., Slaney, C., \& Alda, M. (2014). Nonlinear dynamics of mood regulation in bipolar disorder. Bipolar Disorders, 17, 139-149.

Paluŝ, M. (2008). Bootstrapping multifractals: Surrogate data from random cascades on wavelet dyadic trees. Physical Review Letters, 101, 1234101.

Peng, C.K., Havlin, S., Stanley, H.E., \& Goldberger, A.L. (1995). Quantification of scaling exponents and crossover phenomena in nonstationary heartbeat time series. Chaos, 5, $82-87$.

R Core Team (2021). R: A language and environment for statistical computing. R Foundation for Statistical Computing, Vienna, Austria. URL https://www.R-project.org/

Ratheesh, A., Berk, M., Davey, C.G., McGorry, P.D., \& Cotton, S.M. (2015). Instruments that prospectively predict bipolar disorder - A systematic review. Journal of Affective Disorders, 179, 65-73. 
Schreiber, T., \& Schmitz, A. (2000). Surrogate time series. Physica D, 142, 346-382.

Shannon, C. E. (1948). A mathematical theory of communication. Bell System Technical Journal, 27, 379-423.

Torick, T., \& Mandelkern, M.A. (2013). Multifractal Detrended Fluctuation Analysis of human EEG: Preliminary investigation and comparison with the Wavelet Modulus Maxima Technique. PloS One, 8(7): e68360. Doi:10.1371/journal.pone.0068360

Yamahara, M., Noguchi, T., Okawa, M., \& Yamada, N. (2009). The relationship between subjective sleep disturbance and complexity of 24-hour activity utilizing fractal theory in psychiatric inpatients. Sleep \& Biological Rhythms, 7, 11-16.

\section{ACKNOWLEGEMENTS}

I am pleased to thank Professor Greg Murray from the School of Psychology, Swinburne University, Australia and Dr Tanya Hanstock from the School of Psychological Science, University of Newcastle, Australia for their generosity in providing confidential patient data for me to analyse.

\section{APPENDIX}

\section{A Model for Activity and Heart Rate Data Based on a Stochastic Multiplicative Cascade}

The formulation of this model for activity or heart rate time series, as a representative measure of more general psychomotor and cardiovascular activity, follows closely the mathematics provided by Calvert, Fisher and Mandelbrot (1997). The proofs are contained in the original paper and only the necessary mathematics for the analysis of activity data are presented here. 
Consider a psychomotor or autonomic process, $P(t)$, that evolves over time such that the infinitesimal change at time $t$ is given by $|P(t+d t)-P(t)|=C(t) d t^{\alpha(t)} \cdot \alpha(t)$, the local Hölder exponent, is in general a function of time, and $C(t)$ is either a function of time or a constant. In the case of a diffusion process driven by a Gaussian process with variance $\sigma^{2}$, the infinitesimal step size at time $t$ has variance $\sigma^{2} t$ so that the increment at time $t$ is proportional to $\sigma d t^{0.5}$ in which case $\alpha(t)=0.5$ and $C(t)=\sigma$. More generally, for a fractional Brownian diffusion process we have $\alpha(t)=H, 0<H<1$, where $H$ is the Hurst index, a value not necessarily equal to 0.5 as is the case for the Gaussian process. When $H>0.5$, there are positive correlations between successive values of the time series, so that large values tend to follow large values and vice versa for small values. The time series is persistent. When $H<0.5$, there are negative correlations between successive values of the time series, so that large values tend to follow small values and vice versa for small values. The time series is anti-persistent. When $H=0.5$, successive values of the time series are uncorrelated. In each of these cases, the local Hölder exponent is constant and does not change with time.

We will suppose that psychological and autonomic processes affected by mood fluctuations can be represented by cascaded operations occurring at increasingly finer time scales (Ihlen, 2013). This hierarchical network model is shown in Figure A1 for a binary multiplicative cascade for which each lower level of the hierarchy represents information processing on a successively shorter time scale. Such a process, following the example provided by Calvert et al. (1997), is generated by assuming a unit processing interval [0,1] at stage $k=0$. At stage $k=1$, a binomial process distributes processing capacity so that a proportion $p$ is allocated to the interval $[0,0.5]$ and the remaining proportion $1-p$ is allocated to $[0.5,1]$. At stage $k=2$, the interval $[0,0.5]$ is split into two subintervals $[0,0.25]$ and $[0.25,0.5]$, these subintervals receiving proportions $p$ and $1-p$ of the capacity that was already allocated to [0.0.5]. Similarly, the interval $[0.5,1]$ is split into subintervals $[0.5,0.75]$ and $[0.75,1.0]$ these 
subintervals receiving proportions $p$ and $1-p$ of the capacity allocated to $[0.5,1.0]$, respectively. After two steps the probability allocated to the four subintervals is given by

$$
\begin{gathered}
\operatorname{Pr}[0, .25]=p^{2} \\
\operatorname{Pr}[0.25,0.5]=\operatorname{Pr}[0.5,0.75]=p(1-p), \\
\operatorname{Pr}[0.75,1.0]=(1-p)^{2} .
\end{gathered}
$$

After $k$ steps, the process is binomial with a conservative probability measure

$$
\operatorname{Pr}\left[t, t+2^{-k}\right]=p^{j}(1-p)^{k-j}, j=0, k
$$

In this equation, $t$ is the lower bound of each of the $2^{k}$ subintervals at stage $k$.

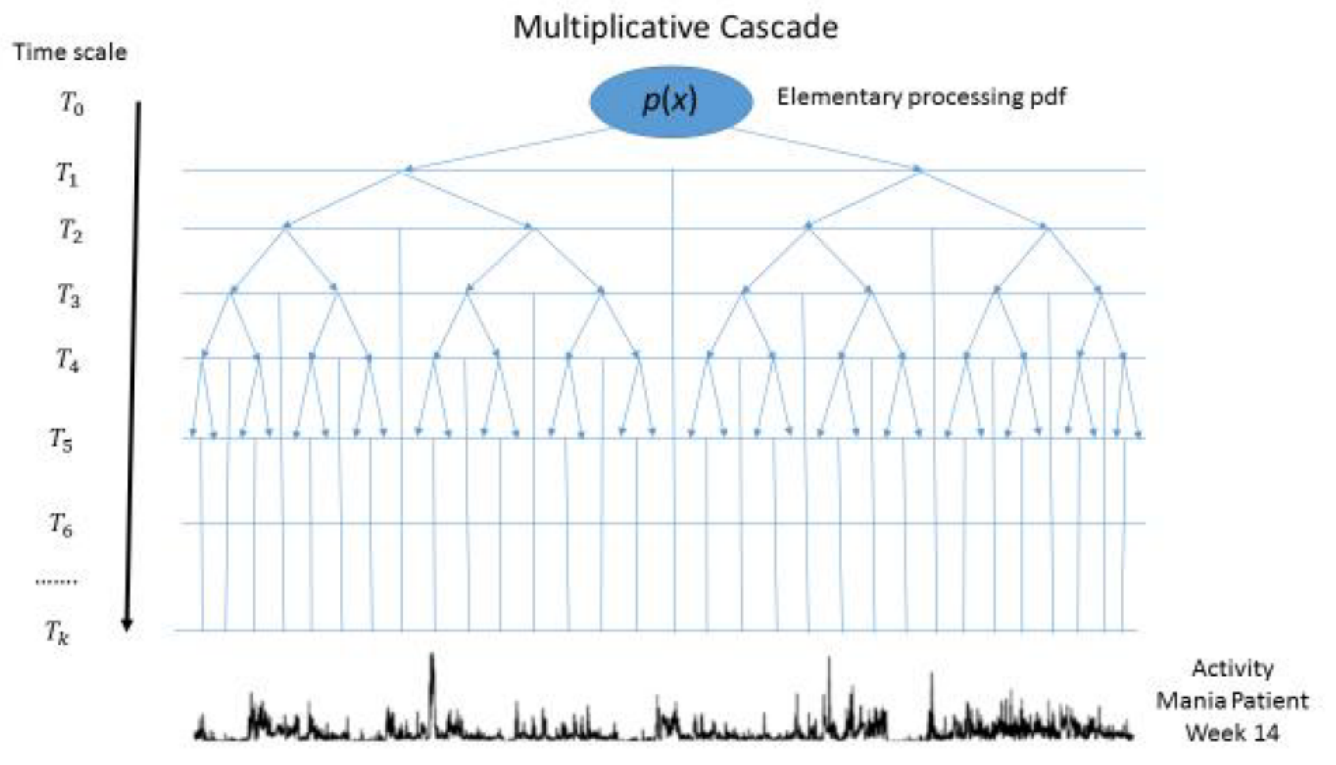

Figure A1. A multiplicative cascade process driven by a pdf, $p(x)$, at increasingly fine binary time scales starting with the complete time interval at $T_{0}$ and increasing the number of intervals by an integer power of two so that at time scale $T_{k}$ the number of intervals is $2^{\mathrm{k}}$. It is assumed that the activity time series shown at the bottom of the graph is generated by a multiplicative cascade in which $p(x)$ is a Gaussian density with mean $\mu$ and variance $\sigma^{2}$.

\section{Multifractal Processes Derived from Multiplicative Cascades with Stochastic}

Flow

A multifractal process obeys nonlinear properties at multiple time scales. A fractal process, on the other hand, considers nonlinear processes that operate on only one time scale. The 
latter is a characteristic feature of all chaotic processes that are sensitively dependent on their initial conditions while remaining relative constrained in their overall dynamics. For example, a tree growing in a pot in a room will always leave some air in the room no matter how many years it is left to grow there! Fractal processes can be distinguished from purely random processes with unstable unconstrained dynamics that essentially fill any space in which they are imbedded, such as air in a room. In this and the following sections we derive expressions for the multifractal spectrum for information processing within a hierarchical multiplicative cascade that is driven by a Gaussian stochastic process.

For a process evolving over a time interval $T$ in a stochastic multiplicative cascade, we assume that its change within the small temporal subinterval of duration, $\Delta t=b^{-k} T$, at the $k$-th stage of a multiplicative cascade with statistically independent between-stage information processing, can be represented by the infinitesimal product:

$$
P\left(t+b^{-k} T\right)-P(t)=p_{1} p_{12} p_{123} \ldots p_{123 \ldots k}
$$

$p_{123 \ldots k}$ is the probability measure associated with any of the $b^{k}$ time subintervals at the $k$-th stage of the cascade.

Define the coarse Hölder exponent of the psychological process $P$ for $t \varepsilon[0, T]$ as

$$
\alpha_{k}(t) \equiv \frac{\log \left\{P\left(t+b^{-k} T\right)-P(t)\right\}}{\log \left(b^{-k}\right)}
$$

By substituting Eq. (A1) into Eq. (A2), the coarse Hölder exponent can be represented by the mean of $k$ identically and independently distributed random variables independently of $t$ as:

$$
\begin{aligned}
\alpha_{k}= & -\frac{1}{k}\left[\log _{b}\left(p_{1}\right)+\log _{b}\left(p_{12}\right)+\log _{b}\left(p_{123}\right)+\cdots+\log _{b}\left(p_{123 \ldots k}\right)\right] \\
= & \frac{1}{k} \sum_{i=1}^{k} V_{i}
\end{aligned}
$$


For sufficiently large $k$, Eq. (A3) satisfies the strong law of large numbers, so that $V_{i}=-\log _{b}\left(p_{1 \ldots i}\right)$. In this case, the random variable for all $t, \alpha_{k}$, converges to $\alpha_{0}=-E\left[\log _{b}\left(p_{1}\right)\right], \alpha_{0}>0$, as $k \rightarrow 0$, where $E[$.$] is the expectation operator and$ $p_{1}$ represents the generic between-stage probability distribution. If $p_{1}$ has finite variance, $\sigma^{2}$, then according to the central limit theorem, as $k \rightarrow \infty, \sqrt{k}\left(\alpha_{k}-\alpha_{0}\right)$ is normally distributed with mean 0 and variance $\sigma^{2}$.

Rather than maintain conservative multiplicative cascade probabilities, the total multiplicative probability in Eq. A1 can be set equal to one under expectation. This allows the product term in Eq. A1 to be premultiplied by a canonical probability associated with the $k$-th stage, $\pi_{123 \ldots k}$, to produce:

$$
\mu_{k}(t)=\pi_{123 \ldots k} p_{1} p_{12} p_{123} \ldots p_{123 \ldots k}
$$

Under such conditions, a stochastic process, $X(t)$, is a multifractal if it satisfies the $q$-th moment expectation equation:

$$
E\left(|X(t)|^{q}\right)=c(q) t^{\tau(q)+1}
$$

where $q$ and $t$ are the singularity moment and time, respectively. $c(q)$ and $\tau(q)$ are scaling functions defined with respect to $q$ (Calvert et al., 1997, p.4).

\section{Computing the Multifractal Spectrum for Continuous Random Variables}

For a continuous multiplicative density, assume that $V_{1}=-\log _{b}$, has density $p(\alpha)$, and let $p_{k}(\alpha)$ be the density of the $k$-th convolution of $p(\alpha)$. According to Theorem 6 of Calvert et al. (1997), the multifractal spectrum for a multiplicative cascade with multipliers satisfying a continuous density $p(\alpha)$ is given by

$$
f(\alpha)=1+\lim _{k \rightarrow \infty} \frac{1}{k} \log _{b}\left[k p_{k}(k \alpha)\right]
$$


For $V_{k}$ distributed as a Gaussian density with mean $\mu$ and variance $\sigma^{2}$,

$$
p_{k}(x)=\frac{1}{\sqrt{2 \pi \sigma^{2}}} \exp \left[-\frac{(x-\mu)^{2}}{2 \sigma^{2}}\right]
$$

Calvert et al. (1997) have shown that

$$
\begin{aligned}
f(\alpha) & =1+\lim _{k \rightarrow \infty} \frac{1}{k} \log _{b}\left[k p_{k}(k \alpha)\right] \\
& =1-\frac{1}{2 \log (b)}\left(\frac{\alpha-\mu}{\sigma}\right)^{2}
\end{aligned}
$$

The value of $\alpha$ that corresponds to the maximum of $f(\alpha)=1$ is $\mu$, the mean of the Gaussian process. The width of $f(\alpha)$ can be obtained by taking the difference between the values of $\alpha$ at the two roots of $f\left(\alpha_{0}\right)=0$. So $\alpha_{0}=\mu \pm \sigma \sqrt{2 \log (b)}$, and the multifractal spectrum width is defined by $K \sigma$ where $K=2 \sqrt{2 \log (b)}$. So, the standard deviation of the Gaussian process is proportional to the width of the multifractal spectrum. We can obtain approximate qualitative estimates of $\mu$ and $\sigma$ by eye-balling the multifractal spectrum produced by the data series. These mathematical derivations are consistent with simulations of a similar multiplicative cascade by Ihlen and Vereijken (2010, Figure A2) as was shown by Figure 2 in the main text. The width of the multifractal spectrum is related to the variance of a lognormal distribution of interaction multipliers.

\section{Continuous Shannon Entropy for the Log Normal Density}

To provide an index associated with the amount of uncertainty associated with any sequence of stimulus events, application is made of Shannon entropy. This measure has a long history starting with the pioneering mathematical work of Shannon (1948) and its applications in various fields of science. As the probability associated with each series of end branches of a 
hierarchical cascade process driven by a Gaussian is the product of independent Gaussian random variables, we take the logarithm of this product as the distribution used to estimate entropy or information capacity. This estimate is easy to obtain in closed form for the Gaussian process, as follows.

The lognormal pdf corresponding to the Gaussian pdf with mean $\mu$ and variance $\sigma^{2}$,

$$
p(x)=\frac{1}{\sqrt{2 \pi} \sigma} \exp \left[-\frac{(x-\mu)^{2}}{\sigma^{2}}\right]
$$

is given by

$$
q(x)=\frac{1}{\sqrt{2 \pi} \sigma x} \exp \left[-\frac{(\ln x-\mu)^{2}}{2 \sigma^{2}}\right]
$$

The continuous Shannon entropy for the lognormal pdf is defined by

$$
\begin{gathered}
E(\mu, \sigma)=-\int_{0}^{\infty} q(x) \ln q(x) d x \\
=-\int_{0}^{\infty} \frac{1}{\sqrt{2 \pi} \sigma x} \exp \left[-\frac{(\ln x-\mu)^{2}}{2 \sigma^{2}}\right] \ln \left\{\frac{1}{\sqrt{2 \pi} \sigma x} \exp \left[-\frac{(\ln x-\mu)^{2}}{2 \sigma^{2}}\right]\right\} d x \\
=\frac{1}{\sqrt{2 \pi} \sigma}\left[I_{1}(\mu, \sigma)+I_{2}(\mu, \sigma)\right]
\end{gathered}
$$

Applying change of variables, we obtain

$$
\begin{gathered}
y=\frac{\ln x-\mu}{\sqrt{2} \sigma} \rightarrow x=\exp (\sqrt{2} \sigma y+\mu) \rightarrow d x=x \sqrt{2} \sigma d y \\
I_{1}(\mu, \sigma)=\int_{0}^{\infty} \frac{\ln (x \sqrt{2 \pi} \sigma)}{x} \exp \left[-\frac{(\ln x-\mu)^{2}}{2 \sigma^{2}}\right] d x
\end{gathered}
$$




$$
\begin{gathered}
=\sqrt{2} \sigma \int_{-\infty}^{\infty}\{\sqrt{2} \sigma y+\mu+\ln (\sqrt{2 \pi} \sigma)\} e^{-y^{2}} d y \\
=\sqrt{2} \sigma[\mu+\ln (\sqrt{2 \pi} \sigma)] \int_{-\infty}^{\infty} e^{-y^{2}} d y+2 \sigma^{2} \int_{-\infty}^{\infty} y e^{-y^{2}} d y \\
=\sqrt{2 \pi} \sigma[\mu+\ln (\sqrt{2 \pi} \sigma)] \\
I_{2}(\mu, \sigma)=\int_{0}^{\infty}\left[\frac{(\ln x-\mu)^{2}}{2 \sigma^{2} x}\right] \exp \left[-\frac{(\ln x-\mu)^{2}}{2 \sigma^{2}}\right] d x \\
=\sqrt{2} \sigma \int_{-\infty}^{\infty} y^{2} e^{-y^{2}} d y \\
=\sigma \sqrt{\frac{\pi}{2}}
\end{gathered}
$$

Substituting $I_{1}(\mu, \sigma)$ and $I_{2}(\mu, \sigma)$ into Eq. (A7) yields

$$
E(\mu, \sigma)=\frac{\sqrt{2 \pi} \sigma[\mu+\ln (\sqrt{2 \pi} \sigma)]+\sigma \sqrt{\frac{\pi}{2}}}{\sqrt{2 \pi} \sigma}
$$

So, the entropy for a log Gaussian pdf is given by

$$
E(\mu, \sigma)=\mu+\frac{\ln \left(2 \pi \sigma^{2}\right)+1}{2}
$$

$E(\mu, \sigma)$ can be computed using estimates of $\mu$ and $\sigma$ obtained by fitting Eq. A6 with $b=2$ to the multifractal spectrum estimated from the activity time series data. The following section shows how this calculation can be performed with time series data.

\section{Analyzing Time Series Data Using Multifractal Detrended Fluctuation}

\section{Analysis}


Multifractal Detrended Fluctuation Analysis (MFDFA) (Kantelhardt, Zschiegner, KoscielnyBunde, Bunde, Havlin \& Stanley, 2002) is a generalization of Detrended Fluctuation Analysis (DFA) that allows for a continuum of fractal or Hurst exponents, as described in the main text. To compute DFA we consider a time series, $x(i), i=1, N$, containing $N$ observations and compare each observation with the average of all observations, leading to the deviation score, $x(i)-\bar{x}$, the difference between the i-th observation of a sequence of $N$ observations and their mean, $\bar{x}$. These deviation scores are added to yield a cumulative deviation score given by

$$
y(k)=\sum_{i=1}^{k}[x(i)-\bar{x}], k=1, N
$$

Interesting properties of the cumulative time sequence, $y(k)$, can be obtained by partitioning $y(k)$ into $N / n$ nonoverlapping subsequences of size $n$, fitting a linear segment to each subsequence to produce $y(n, k)$, and then computing the average fluctuation of the integrated series about the trend line as follows (Peng, Havlin, Stanley \& Goldberger, 1995, Equation 1):

$$
F(n)=\sqrt{\frac{1}{N} \sum_{k=1}^{N}[y(k)-y(n, k)]^{2}}
$$

MFDFA is a generalization of DFA to include processes that result from a continuum of Hurst exponents. The cumulative time series subsequence is divided into $N_{s} \equiv \operatorname{int}(N / s)$ nonoverlapping segments, each of size $s$, starting at the first observation in the series. This procedure is repeated, starting from the last observation of the series and working backwards to ensure that the last observation of the series is included in the calculations. So, the complete analysis uses $2 N_{s}$ segments from the original subsequence. Further partitioning of 
the series is obtained by using successively smaller segment sizes, the number of segments in any given partition being usually twice that of the preceding partition.

For segment $v, v=1, N_{s}$, values starting from the first observation are fit with a polynomial $\hat{y}_{v}(i)$ estimated by minimising the total sum of squared deviations. The order of this polynomial, $m$, is small and usually restricted to accommodate no more than cubic trends. In most applications, the multifractal spectrum does not depend on the choice of $m$. In the calculations performed in this paper, $m=3$, a cubic polynomial. The minimum value of the segment size, $s$, equals $m+2$, the smallest number of values required for a nontrivial fit of an $m$-order polynomial to the segment.

The residual variance for segment $v$ is obtained by first computing the difference between observed and fitted values using the best-fitting polynomial, squaring this difference, and computing the average squared difference to yield a fluctuation function defined by

$$
F^{2}(s, v)=\frac{1}{s} \sum_{i=1}^{s}\left\{y[(v-1) s+i]-\hat{y}_{v}(i)\right\}^{2}
$$

where $s$ is the segment size. The corresponding residual variance using the last observation of each subsequence is given by

$$
F^{2}(s, v)=\frac{1}{s} \sum_{i=1}^{s}\left\{y\left[N-\left(v-N_{s}\right) s+i\right]-\hat{y}_{v}(i)\right\}^{2}
$$

In the generalized version of DFA, the variance function is averaged over all the segments to obtain the $q$-th order fluctuation function defined by

$$
F_{q}(s)=\left\{\frac{1}{2 N_{s}} \sum_{v=1}^{2 N_{s}}\left[F^{2}(s, v)\right]^{q / 2}\right\}^{1 / q}
$$


where $q$ is any real number other than 0 . As a special case, the standard form of the basic DFA relationship is obtained when $q=2$.

$$
D F A \stackrel{\text { def }}{=} F_{2}(s)=\left\{\frac{1}{2 N_{s}} \sum_{v=1}^{2 N_{s}} F^{2}(s, v)\right\}^{1 / 2}
$$

The temporal scaling of the fluctuation function is obtained by computing $F_{q}(s)$ for several values of $s$ and estimating the exponent, $h(q)$, assuming that $F_{q}(s)$ is a power function of $s$ as shown by

$$
F_{q}(s)=s^{h(q)}
$$

$h(q)$ is a generalization of the Hurst exponent, $H=h(2)$, obtained when $q=2$. The Hurst exponent and its generalized version provide information about temporal fluctuations in a time series on all time scales.

Complex fluctuations in time series can be evaluated by estimating the generalized Hurst exponent, $h(q)$, for various values of $q$. If $h(q)$ does not depend on $q$, the time series is defined as monofractal, implying that all types of fluctuations, large and small, scale in the same way. If $h(q)$ varies with $q$, the time series contains a complex mixture of fluctuations leading to multifractality. Positive values of $q$ reflect the effect of large-scale fluctuations, whereas negative values of $q$ result from fluctuations with small variance.

Kandelhardt et al. (2002) suggest that the above method can be used to perform multifractal analysis provided negative generalized Hurst functions can also be accommodated. This can be achieved by summing $y(k)$ values defined in Eq. (A9), as follows:

$$
Y(i)=\sum_{k=1}^{i}[y(k)-\bar{y}]
$$


When these summed values are used instead of the $y(i)$ in Eqs. (A11) and (A12), the only change is the addition of 1 to the power in the power law expressed in Eq. (A15) to obtain

$$
F_{q}(s)=s^{h(q)+1}
$$

This modification allows the fluctuation function to represent scaling behaviour when $h(q)$ is negative but greater than -1 (Kandelhardt et al., 2002).

As shown by Kandelhardt et al. (2002), $h(q)$ is related to the classical multifractal exponent function $\tau(q)$ obtained from a box-counting algorithm by

$$
\tau(q)=q h(q)-1
$$

A box counting algorithm allows the analysis of complex patterns, in this case temporal, by dividing the data into increasingly smaller subsets so that a suitable scaling factor can be determined. In this way a measure of the complexity of the time series can be obtained that is independent of the scale of measurement. Such measures include the fractal dimensionality, most often a fractional number that determines how successive values of a time series relate to each other. MFDFA obviates the need to employ such detailed box counting methods in multifractal analysis.

The singularity spectrum for a multifractal, otherwise known as the multifractal spectrum, is obtained by plotting $f(\alpha)$ against $\alpha$, where

$$
\alpha=\frac{d \tau(q)}{d q}
$$

and

$$
f(\alpha)=q \alpha-\tau(q)
$$

Equations (A18) and (A19) satisfy the requirements of a Legendre transform. $\alpha$ is the $q$-order singularity strength or Hölder exponent. A singularity is a point on a function for which the 
first derivative might not exist, a characteristic feature of noncontinuous fractal and multifractal processes. For best outcomes, including the valid application of a conventional surrogate test for nonlinearity without the need to compute complicated multifractal surrogates (Palus, 2008), the range of $q$ should be between -5 and +5 . This range of $q$ values was used in all the data analyses reported in this paper.

\section{Software for Multifractal Detrended Fluctuation Analysis}

The following software summary is based on the algorithm described by Ihlen (2012), who provided a MATLAB routine for performing the calculations. This routine was translated into commonly used statistical software package, $R$ (R Core Team, 2021), augmented by the nonlinearTseries package (Garcia, 2021) to compute phase-randomised surrogate series that preserved all the linear properties, mean, variance and autocorrelation function, of the original RT series, and the DEoptim package (Mullen, Ardia, Gil, Windover, \& Cline, 2011) to compute parameter estimates for a nonlinear function using Differential Evolution.

The calculations were performed using several data window sizes ranging from $2^{3}=8$, to the power of two that is no greater than $20 \%$ of the number of observations in the time series. The order of the polynomial fit to the cumulated data during detrending (Eq. A11) can be either linear, quadratic or cubic, a value set in advance prior to the analysis. In all data analyses reported in this paper a cubic polynomial was used for detrending, a compromise between the effects of a number of short-term influences on performance, such as attention fluctuations and motor fatigue, and model parsimony. The computer program returns the fluctuation function variance (Eq. 13), the generalized Hurst exponent (Eq. 15), the multifractal exponent (Eq. A17), and the abscissae and ordinates of the multifractal spectrum (Eqs. A18 and A19, respectively). 